\title{
Formation of Finite-Time Singularities in the 3D Axisymmetric Euler Equations: A Numerics Guided Study*
}

\author{
Guo Luo $^{\dagger}$ \\ Thomas Y. Hou
}

\begin{abstract}
Whether the three-dimensional incompressible Euler equations can develop a singularity in finite time from smooth initial data is one of the most challenging problems in mathematical fluid dynamics. This work attempts to provide an affirmative answer to this long-standing open question, by first describing a class of potentially singular solutions to the Euler equations numerically discovered in axisymmetric geometries, and then by presenting evidence from rigorous analysis that strongly supports the existence of such singular solutions. The initial data leading to these singular solutions possess certain special symmetry and monotonicity properties, and the subsequent flows are assumed to satisfy a periodic boundary condition along the axial direction and a no-flow, free-slip boundary condition on the solid wall. The numerical study employs a hybrid 6th-order Galerkin/finite difference discretization of the governing equations in space and a 4th-order Runge-Kutta discretization in time, where the emerging singularity is captured on specially designed adaptive (moving) meshes that are dynamically adjusted to the evolving solutions. With a maximum effective resolution of over $\left(3 \times 10^{12}\right)^{2}$ near the point of the singularity, the simulations are able to advance the solution to a point that is asymptotically close to the predicted singularity time, while achieving a pointwise relative error of $O\left(10^{-4}\right)$ in the vorticity vector and obtaining a $\left(3 \times 10^{8}\right)$-fold increase in the maximum vorticity. The numerical data are checked against all major blowup/nonblowup criteria, including Beale-Kato-Majda, ConstantinFefferman-Majda, and Deng-Hou-Yu, to confirm the validity of the singularity. A close scrutiny of the data near the point of the singularity also reveals a self-similar structure in the blowup, as well as a one-dimensional model which is seen to capture the essential features of the singular solutions along the solid wall, and for which existence of finite-time singularities can be established rigorously.
\end{abstract}

Key words. 3D axisymmetric Euler equations, finite-time blowup

AMS subject classifications. 35Q31, 76B03, 65M60, 65M06, 65M20

DOI. $10.1137 / 19 \mathrm{M} 1288061$

\section{Contents}

I Introduction

2 Description of the Problem

*Published electronically November 6, 2019. This paper originally appeared in Multiscale Modeling and Simulation, Volume 12, Number 4, 2014, pages 1722-1776, under the title "Toward the Finite-Time Blowup of the 3D Axisymmetric Euler Equations: A Numerical Investigation."

https://doi.org/10.1137/19M1288061

Funding: This work was supported in part by NSF FRG grant DMS-1159138 and NSF grants DMS-1613861, DMS-1907977, and DMS-1912654.

$\dagger$ Department of Mathematics, City University of Hong Kong, Kowloon Tong, Hong Kong (guoluo@cityu.edu.hk).

${ }^{\ddagger}$ Applied \& Computational Mathematics, California Institute of Technology, MC 9-94, Pasadena, CA 91125 (hou@cms.caltech.edu). 
3 Outline of the Numerical Method $\quad \mathbf{8 0 0}$

3.1 The Adaptive (Moving) Mesh Algorithm . . . . . . . . . . . . . . . 800

3.2 The B-Spline-Based Galerkin Poisson Solver . . . . . . . . . . . . . 802

3.3 The Overall Algorithm . . . . . . . . . . . . . . . . . . . . . . . 804

4 Numerical Results $\quad \mathbf{8 0 5}$

4.1 Effectiveness of the Adaptive Mesh _ . . . . . . . . . . . . 805

4.2 First Sign of Singularity . . . . . . . . . . . . . . . 807

4.3 Resolution Study . . . . . . . . . . . . . . . . . . . . 808

4.3.1 Code Validation on Test Problems . . . . . . . . . . . . 810

4.3.2 Resolution Study on Transformed Primitive Variables . . . . . 810

4.3.3 Resolution Study on Vorticity Vector . . . . . . . . . . . . 812

4.3.4 Resolution Study on Global Quantities . . . . . . . . . . . . 812

4.3.5 Resolution Study in Time . . . . . . . . . . . . . . . . 815

4.4 Asymptotic Scaling Analysis I: Maximum Vorticity . . . . . . . . . 815

4.5 Asymptotic Scaling Analysis II: Vorticity Moments . . . . . . . . . . 819

4.6 Vorticity Direction and Spectral Dynamics _ . . . . . . . . . . . 820

4.7 Locally Self-Similar Structure _ . . . . . . . . . . . . . . . . . 823

4.7.1 Existence of Self-Similar Neighborhood . . . . . . . . . . 825

4.7 .2 Existence of Self-Similar Profiles . . . . . . . . . . . . 826

4.7 .3 Self-Similar Analysis . . . . . . . . . . . . . . 827

4.8 Understanding the Blowup . . . . . . . . . . . . . . . . 828

4.9 A 1D Model . . . . . . . . . . . . . . . . . . . . . . . . 829

5 Conclusions and Future Work $\quad 830$

$\begin{array}{ll}\text { Acknowledgments } & 832\end{array}$

$\begin{array}{ll}\text { References } & 832\end{array}$

I. Introduction. The celebrated three-dimensional (3D) incompressible Euler equations in fluid dynamics describe the motion of ideal incompressible flows in the absence of external forcing. First written down by Leonhard Euler in 1757, these equations take the form

$$
u_{t}+u \cdot \nabla u=-\nabla p, \quad \nabla \cdot u=0,
$$

where $u=\left(u_{1}, u_{2}, u_{3}\right)^{T}$ is the $3 \mathrm{D}$ velocity vector of the fluid and $p$ is the scalar pressure. Utilized extensively by physicists to model ocean currents, weather patterns, and other fluid related phenomena, the 3D Euler equations encompass a rich mathematical theory, for which the interested readers may consult the excellent surveys $[2,24,34]$ and the references therein. This paper primarily concerns the existence or nonexistence of globally (in time) regular, finite-energy solutions to the 3D Euler equations, which is regarded as one of the most fundamental yet most challenging problems in mathematical fluid dynamics. ${ }^{1}$

The significance of the global regularity or finite-time blowup of (1.1) manifests itself in several ways. Mathematically, the question has remained open for over 250

\footnotetext{
${ }^{1}$ The existence of infinite-energy, finite-time blowup solutions to the 3D Euler equations has long been known; see, for example, [35] and the references therein.
} 
years and has a close connection to the Clay Millennium Prize Problem on the NavierStokes equations [31]. Physically, the formation of a singularity in inviscid (Euler) flows may signify the onset of turbulence in viscous (Navier-Stokes) flows, and it may provide a mechanism for energy transfer to small scales. Numerically, the resolution of nearly singular flows requires special numerical treatments, which presents a great challenge to computational scientists.

The difficulty associated with the regularity properties of the 3D Euler equations can be best understood via the so-called vorticity-stream formulation,

$$
\omega_{t}+u \cdot \nabla \omega=\omega \cdot \nabla u
$$

which can be derived from (1.1) by applying the curl operator $\nabla \times$ on both sides of the equation. Here, in (1.2), $\omega=\nabla \times u$ is the vorticity vector of the fluid, and $u$ is related to $\omega$ via a Biot-Savart law. In free space $\mathbb{R}^{3}$, the Biot-Savart law takes the form

$$
u(x)=\frac{1}{4 \pi} \int_{\mathbb{R}^{3}} \frac{x-y}{|x-y|^{3}} \times \omega(y) d y,
$$

while in domains with solid boundaries, representations of similar natures can also be written down. Straightforward calculations then reveal that $\nabla u$ is related to $\omega$ via a singular integral operator, and standard estimates from the theory of such operators imply (see, for example, [66])

$$
\|\omega\|_{L^{p}} \leq\|\nabla u\|_{L^{p}} \leq C_{p}\|\omega\|_{L^{p}}, \quad 1<p<\infty .
$$

This heuristic argument shows that the vortex stretching term $\omega \cdot \nabla u$ scales formally like $\omega^{2}$, and hence it indeed has the potential to generate a finite-time singularity. ${ }^{2}$

Considerable efforts have been devoted to the study of the regularity properties of the 3D Euler equations. As suggested by (1.2)-(1.3), the main difficulty in the analysis lies in the presence of the nonlinear vortex stretching term and the lack of a regularization mechanism, which implies that even the local well-posedness of the equations can only be established for sufficiently smooth initial conditions (see, for example, [49]). Despite these difficulties, a few important partial results $[4,62,32,64,25,26,36]$ have been obtained over the years, which have led to improved understanding of the regularity properties of the 3D Euler equations. More specifically, the celebrated theorem of Beale, Kato, and Majda [4] and its variants [32,64] characterize the regularity of the 3D Euler equations in terms of the maximum vorticity, asserting that a smooth solution $u$ of (1.1) blows up at $t=T$ if and only if

$$
\int_{0}^{T}\|\omega(\cdot, t)\|_{L^{\infty}} d t=\infty
$$

The nonblowup criterion of Constantin, Fefferman, and Majda [25] focuses on the geometric aspects of Euler flows instead, and it asserts that there can be no blowup if the velocity field $u$ is uniformly bounded and the vorticity direction $\xi=\omega /|\omega|$ is sufficiently "well behaved" (Lipschitz continuous) near the point of the maximum vorticity. The theorem of Deng, Hou, and Yu [26] is similar in spirit to the ConstantinFefferman-Majda criterion, but confines the analysis to localized vortex line segments.

Besides the analytical results mentioned above, which primarily focus on mathematical conditions under which (smooth) solutions of the 3D Euler equations remain

\footnotetext{
${ }^{2}$ It does not, however, guarantee the existence of a finite-time singularity, since no such estimates as $\|\omega \cdot \nabla u\|_{L^{p}} \geq C\|\omega\|_{L^{p}}\|\nabla u\|_{L^{p}}$ exist.
} 
globally regular, considerable efforts have also been devoted to the (numerical) search of a finite-time Euler singularity. Representative work in this direction include [38, 63], which studied Euler flows with swirls in axisymmetric geometries; the famous computation of Kerr and his collaborators [50, 10, 51], which studied Euler flows generated by a pair of perturbed antiparallel vortex tubes; and the viscous simulations of [7], which studied the 3D Navier-Stokes equations using Kida's high-symmetry initial conditions. Other relevant work includes [12,65], which studied axisymmetric Euler flows with complex initial data and reported singularities in the complex plane. A more comprehensive list of interesting numerical results can be found in the recent review [34].

Although finite-time singularities were frequently reported in numerical studies of the 3D Euler equations, most such singularities turned out to be false predictions, as a result of either inadequately resolved computations or incautious data analysis practices (more to follow on this topic in section 4.4). Indeed, by exploiting the analogy between the two-dimensional (2D) Boussinesq equations and the 3D axisymmetric Euler equations away from the symmetry axis, E and Shu [27] studied the potential development of finite-time singularities in the 2D Boussinesq equations, with initial data completely analogous to those of $[38,63]$. They found no evidence for singular solutions, indicating that the "blowups" reported by $[38,63]$, which were located away from the axis, are likely numerical artifacts. Likewise, Hou and Li [44] repeated the computation of [50] with higher resolutions, in an attempt to reproduce the singularity observed in that study. Despite some ambiguity in the interpretation of the initial data used by [50], they managed to advance the solution up to $t=19$, which is beyond the singularity time $T=18.7$ alleged by [50]. By using newly developed analytic tools based on rescaled vorticity moments, Kerr also confirmed in a recent study [51] that solutions computed from initial data analogous to that used in [50] eventually converge to superexponential growth, and hence are unlikely to lead to a singularity. In a later work, Hou and $\mathrm{Li}$ [45] also repeated the computation of [7] and found that the singularity reported by [7] is likely an artifact due to insufficient resolution.... To summarize, the existing numerical studies do not seem to provide convincing evidence to support the existence of a finite-time Euler singularity, and the question of whether initially smooth solutions to (1.1) can blow up in finite time remains wide open.

By focusing on solutions with rotational symmetry and other special properties, we have discovered, through careful numerical studies, a class of potentially singular solutions to the 3D axisymmetric Euler equations in a radially bounded, axially periodic cylinder (see (2.1)-(2.2) below). The reduced computational complexity in the cylindrical geometry greatly facilitates the computation of the singularity. With a specially designed adaptive mesh, we are able to achieve a maximum mesh resolution of over $\left(3 \times 10^{12}\right)^{2}$ near the point of the singularity; this allows us to compute the vorticity vector with four digits of accuracy throughout the simulations, and to observe a $\left(3 \times 10^{8}\right)$-fold amplification in the maximum vorticity. The numerical data are checked against all major blowup/nonblowup criteria, including Beale-Kato-Majda, Constantin-Fefferman-Majda, and Deng-Hou-Yu, to confirm the validity of the singularity. A close scrutiny of the data near the point of the singularity also reveals a self-similar structure in the blowup, as well as a one-dimensional (1D) model which is seen to capture the essential features of the singular solutions along the solid wall, and for which existence of finite-time singularities can be established rigorously. Our numerical method makes explicit use of the special symmetries built in the blowing-up 
solutions, which eliminates symmetry-breaking perturbations and facilitates a stable computation of the singularity.

The main features of the potentially singular solutions that we discover are summarized as follows. The point of the potential singularity, which is also the point of the maximum vorticity, is always located at the intersection of the solid boundary $r=1$ and the symmetry plane $z=0$. It is a stagnation point of the flow, as a result of the special odd-even symmetries of the flow along the axial direction and the no-flow, free-slip boundary condition on the solid wall (see (2.3)). The vanishing velocity field at this point could have positively contributed to the formation of the singularity, given the potential regularizing effect of convection as observed by [43, 42]. When viewed in the meridian plane, the point of the potential singularity is a hyperbolic saddle of the flow, where the axial flow along the solid boundary marches toward the symmetry plane $z=0$ and the radial flow marches toward the symmetry axis $r=0$ (see Figure 11(a)). The axial flow brings together vortex lines near the solid boundary $r=1$ and destroys the geometric regularity of the vorticity vector near the symmetry plane $z=0$, violating the Constantin-Fefferman-Majda and Deng-Hou-Yu geometric nonblowup criteria, and hence leading to the breakdown of the smooth vorticity field.

The asymptotic scalings of the various quantities involved in the potential finitetime blowup are summarized as follows. Near the predicted singularity time $t_{s}$, the scalar pressure and the velocity field remain uniformly bounded, while the maximum vorticity blows up like $O\left(t_{s}-t\right)^{-\gamma}$ where $\gamma$ roughly equals $\frac{5}{2}$. Near the point of the potential singularity, namely, the point of the maximum vorticity, the radial and axial components of the vorticity vector grow roughly like $O\left(t_{s}-t\right)^{-5 / 2}$ while the angular vorticity grows like $O\left(t_{s}-t\right)^{-1}$. The nearly singular solution has a locally self-similar structure in the meridian plane near the point of the singularity, with a rapidly collapsing support whose diameter scales roughly like $O\left(t_{s}-t\right)^{3}$. When viewed in $\mathbb{R}^{3}$, this corresponds to a thin tube on the symmetry plane $z=0$ evolving around the ring $r=1$, where the radius of the tube shrinks to zero as the singularity forms.

We emphasize that the 3D axisymmetric Euler equations (2.1) are different from their free-space counterpart (1.1), in that they have a constant of motion that is not present in the nonsymmetric case [59]. In addition, it is well known that the choice of the boundary conditions (periodic vs. no-flow) has a nontrivial impact on the qualitative behavior of the solutions of the Euler equations, especially near the solid boundaries $[2,24]$. In view of these differences and the fact that the singularity we discover lies right on the boundary, we stress that the work described in this paper is not directly relevant to the Clay Millennium Prize Problem on the NavierStokes equations, which is posed either in free space or on periodic domains [31]. ${ }^{3}$ Rather, it should be viewed as an attempt at the understanding of the effect of solid boundaries in the creation of small scales and, in the case of zero viscosity, the creation of singularities in incompressible flows.

It is also worth mentioning that since the publication of our original work on the current title in $[57,58]$, great interest has been generated in the field, as a result of which a number of important developments have been subsequently witnessed. In [16], a $2 \mathrm{D}$ incompressible active scalar equation which has the same scaling as the inviscid surface quasi-geostrophic (SQG) equation is proposed and studied, along with other

\footnotetext{
${ }^{3}$ Indeed, according to the partial regularity result of Caffarelli, Kohn, and Nirenberg [11], any finite-time singularity of the 3D axisymmetric Navier-Stokes equations, if it exists, must lie on the symmetry axis.
} 
well-known equations in fluid dynamics. It is shown that this active scalar admits solutions that blow up in finite time, which seems to be the first known result along this direction. In [54], an initial condition possessing symmetry properties similar to those proposed by $[57,58]$ is constructed for the $2 \mathrm{D}$ Euler equations on a disk. It leads to a smooth solution for which the gradient of vorticity exhibits double exponential growth - the fastest possible growth rate - in time for all times, thereby solving a longstanding open problem in the theory of $2 \mathrm{D}$ Euler equations. In [21], a simplified $1 \mathrm{D}$ model that captures the essential features of the 3D Euler blowup scenario is proposed and studied. For a certain class of initial data that possesses symmetry properties similar to those considered in $[57,58]$, it is shown that a singularity forms in finite time, thus providing further supporting evidence for the discoveries of our study. In [53], a family of models that interpolates between the 2D Euler equations and the inviscid SQG equation is proposed and studied. For initial data consisting of disjoint, smoothly bounded vortex patches, it is shown that the 2D Euler solutions remain globally regular, while any model that is slightly more singular than the 2D Euler admits solutions that develop a singularity in finite time. These singular solutions, again, originate from initial data that possess symmetry properties similar to those considered in $[57,58]$, and their loss of regularity in finite time relies crucially on the presence of a solid boundary, much like what has been observed in our study and [54]. Similar studies have also been carried out for the 2D inviscid Boussinesq equations, which are known to be qualitatively similar to the 3D axisymmetric Euler equations away from the symmetry axis and which, therefore, are expected to admit solutions with similar singular behaviors. The related results, together with other recent progress, can be found in the excellent surveys [55, 52].

The blowup results described in the above-mentioned work primarily concern (1D or $2 \mathrm{D}$ ) model equations that provide some form of approximations to the original 3D Euler or 2D Boussinesq equations near the point of the hypothetical singularity. In a series of recent studies $[28,29]$, a different line of attack is followed, where the original 2D Boussinesq and 3D (axisymmetric) Euler equations are studied in a cornered domain, and where the existence of finite-time singularities is explicitly established for a class of strong, locally scale-invariant solutions (see also [30] for a related study that follows a different philosophy). While these results are interesting and significant in their own right, we remark that they do not seem to be directly related to the blowup scenario described here, and thus may not be viewed as rigorous justifications of the latter.

The rest of this paper is devoted to the study of the potentially singular solutions of the 3D Euler equations and is organized as follows. Section 2 briefly reviews the 3D Euler equations in axisymmetric form and defines the problem to be studied, while section 3 describes the numerical method to be used. After examining the numerical data in great detail and presenting evidence supporting the existence of a finite-time singularity in section 4 , we conclude the paper in section 5 , with a brief discussion on potential implications of our findings and a description on possible directions for future explorations.

2. Description of the Problem. The 3D Euler equations (1.1) with axial symmetry can be conveniently described in the so-called vorticity-stream form (cf. (1.2)). To derive these equations, recall first that in cylindrical coordinates $(r, \theta, z)$, an axisymmetric flow $u$ can be described by the decomposition

$$
u(r, z)=u^{r}(r, z) e_{r}+u^{\theta}(r, z) e_{\theta}+u^{z}(r, z) e_{z},
$$


where $e_{r}=(\cos \theta, \sin \theta, 0)^{T}, e_{\theta}=(-\sin \theta, \cos \theta, 0)^{T}$, and $e_{z}=(0,0,1)^{T}$ are coordinate axes. The vorticity vector $\omega=\nabla \times u$ has a similar representation,

$$
\begin{gathered}
\omega(r, z)=\omega^{r}(r, z) e_{r}+\omega^{\theta}(r, z) e_{\theta}+\omega^{z}(r, z) e_{z}, \\
\omega^{r}=-u_{z}^{\theta}, \quad \omega^{\theta}=u_{z}^{r}-u_{r}^{z}, \quad \omega^{z}=\frac{1}{r}\left(r u^{\theta}\right)_{r},
\end{gathered}
$$

where for simplicity we have used subscripts to denote partial differentiations. The incompressibility condition $\nabla \cdot u=0$ implies the existence of a (vector) stream function

$$
\psi(r, z)=\psi^{r}(r, z) e_{r}+\psi^{\theta}(r, z) e_{\theta}+\psi^{z}(r, z) e_{z},
$$

which, without loss of generality, can be assumed to satisfy the divergence-free condition $\nabla \cdot \psi=0$ and for which there hold the representations

$$
u=\nabla \times \psi, \quad \omega=\nabla \times(\nabla \times \psi)=-\Delta \psi .
$$

Taking the $\theta$-components of the velocity equation (1.1), the vorticity equation (1.2), and the Poisson equation $-\Delta \psi=\omega$ gives an alternative formulation of the $3 \mathrm{D}$ Euler equations $[59,43]$

$$
\begin{aligned}
u_{1, t}+u^{r} u_{1, r}+u^{z} u_{1, z} & =2 u_{1} \psi_{1, z}, \\
\omega_{1, t}+u^{r} \omega_{1, r}+u^{z} \omega_{1, z} & =\left(u_{1}^{2}\right)_{z}, \\
-\left[\partial_{r}^{2}+(3 / r) \partial_{r}+\partial_{z}^{2}\right] \psi_{1} & =\omega_{1},
\end{aligned}
$$

where $u_{1}=u^{\theta} / r, \omega_{1}=\omega^{\theta} / r$, and $\psi_{1}=\psi^{\theta} / r$ are transformed angular velocity, vorticity, and stream functions, respectively. ${ }^{4}$ The radial and axial components of the velocity can be recovered from $\psi_{1}$ as

$$
u^{r}=-r \psi_{1, z}, \quad u^{z}=2 \psi_{1}+r \psi_{1, r}
$$

for which the incompressibility condition

$$
\frac{1}{r}\left(r u^{r}\right)_{r}+u_{z}^{z}=0
$$

is satisfied automatically. As shown by [56], $\left(u^{\theta}, \omega^{\theta}, \psi^{\theta}\right)$ must all vanish at $r=0$ if $u$ is a smooth velocity field. Thus $\left(u_{1}, \omega_{1}, \psi_{1}\right)$ are well defined as long as the corresponding solution to (1.1) remains smooth. The reason we choose to work with the transformed variables $\left(u_{1}, \omega_{1}, \psi_{1}\right)$ instead of the original variables $\left(u^{\theta}, \omega^{\theta}, \psi^{\theta}\right)$ is that the equations satisfied by the latter,

$$
\begin{aligned}
u_{t}^{\theta}+u^{r} u_{r}^{\theta}+u^{z} u_{z}^{\theta} & =-\frac{1}{r} u^{r} u^{\theta}, \\
\omega_{t}^{\theta}+u^{r} \omega_{r}^{\theta}+u^{z} \omega_{z}^{\theta} & =\frac{2}{r} u^{\theta} u_{z}^{\theta}+\frac{1}{r} u^{r} \omega^{\theta}, \\
-\left[\Delta-\left(1 / r^{2}\right)\right] \psi^{\theta} & =\omega^{\theta},
\end{aligned}
$$

have a formal singularity at $r=0$, which is inconvenient to work with numerically.

We shall numerically solve the transformed equations (2.1) on the cylinder

$$
D(1, L)=\{(r, z): 0 \leq r \leq 1,0 \leq z \leq L\}
$$

\footnotetext{
${ }^{4}$ These variables should not be confused with the components of the velocity, vorticity, and stream vector functions.
}

Copyright $\odot$ by SIAM. Unauthorized reproduction of this article is prohibited. 
with the initial condition

$$
u_{1}^{0}(r, z)=100 \mathrm{e}^{-30\left(1-r^{2}\right)^{4}} \sin \left(\frac{2 \pi}{L} z\right), \quad \omega_{1}^{0}(r, z)=\psi_{1}^{0}(r, z)=0 .
$$

The solution is subject to a periodic boundary condition in $z$,

$$
u_{1}(r, 0, t)=u_{1}(r, L, t), \quad \omega_{1}(r, 0, t)=\omega_{1}(r, L, t), \quad \psi_{1}(r, 0, t)=\psi_{1}(r, L, t)
$$

and a no-flow, free-slip boundary condition on the solid wall $r=1$ :

$$
\psi_{1}(1, z, t)=0 .
$$

The pole condition

$$
u_{1, r}(0, z, t)=\omega_{1, r}(0, z, t)=\psi_{1, r}(0, z, t)=0
$$

is also enforced at the symmetry axis $r=0$, which ensures the smoothness of the solution.

The initial condition (2.2a) describes a purely rotating eddy in a periodic cylinder, and it satisfies special odd-even symmetries at the planes $z_{i}=\frac{i}{4} L, i=0,1,2,3$. Specifically, $u_{1}^{0}$ is even at $z_{1}, z_{3}$, it is odd at $z_{0}, z_{2}$, and $\omega_{1}^{0}$ and $\psi_{1}^{0}$ are both odd at all $z_{i}$ 's. These symmetry properties are preserved by equations (2.1), so instead of solving the problem $(2.1)-(2.2)$ on the entire cylinder $D(1, L)$, it suffices to consider the problem on the quarter cylinder $D\left(1, \frac{1}{4} L\right)$, with the periodic boundary condition $(2.2 \mathrm{~b})$ replaced by appropriate symmetry boundary conditions. It is also interesting to notice that the boundaries of $D\left(1, \frac{1}{4} L\right)$ behave like "impermeable walls":

$$
u^{r}=-r \psi_{1, z}=0 \quad \text { on } \quad r=1, \quad u^{z}=2 \psi_{1}+r \psi_{1, r}=0 \quad \text { on } \quad z=0, \frac{1}{4} L,
$$

which is a consequence of the no-flow boundary condition (2.2c) and the odd symmetry of $\psi_{1}$.

3. Outline of the Numerical Method. The potential formation of a finite-time singularity from the initial condition (2.2a) makes the numerical solution of the initialboundary value problem (2.1)-(2.2) a challenging and difficult task. In this section, we describe a special mesh adaptation strategy (section 3.1) and a B-spline-based Galerkin Poisson solver (section 3.2), which are essential to the accurate computation of the nearly singular solutions. The overall algorithm is outlined in section 3.3.

3.I. The Adaptive (Moving) Mesh Algorithm. Singularities (blowups) are abundant in mathematical models of physical systems. Examples include the semilinear parabolic equations describing the blowup of the temperature of a reacting medium, such as a burning gas [33], the nonlinear Schrödinger equations describing the selffocusing of electromagnetic beams in a nonlinear medium [60], and the aggregation equations describing the concentration of interacting particles [48]. Often, singularities occur on increasingly small length and time scales, which necessarily requires some form of mesh adaptation. Further, finite-time singularities usually evolve in a "self-similar" manner when singularity time is approached. An adaptive mesh designed for singularity detection must correctly capture these behaviors when applied to problems that are known to admit singular solutions.

Several methods have been proposed to capture (self-similar) singularities in numerical computations. In [60], a dynamic rescaling algorithm is used to solve the 
cubic Schrödinger equation. The main advantage of the method is that the rescaled equation is nonsingular and the rescaled variable is uniformly bounded in appropriate norms. The disadvantage is that the fixed-sized mesh is spread apart by rescaling, so accuracy is inevitably lost away from the singularity.

In [5], a rescaling algorithm is proposed for the numerical solution of the semilinear heat equation, based on the idea of adaptive mesh refinement [6]. The method repeatedly refines the mesh in the "inner" region of the singularity, while rescaling the solution on refined meshes so that it remains uniformly bounded. The main advantage of the method is that it achieves uniform accuracy across the entire computational domain and is applicable to more general problems. The disadvantage is that it requires a priori knowledge of the singularity, and is not easily adaptable to elliptic equations (especially in multiple space dimensions) due to the use of irregular (nested) mesh.

The moving mesh method [47] provides a very general framework for mesh adaptation and has been applied in various contexts, for example, in the solution of the semilinear heat equation [9] and the solution of the nonlinear Schrödinger equation [8]. The main idea of the method is to construct the mesh based on a certain equidistribution principle, for example, the equipartition of the arc length function. In one space dimension this completely determines the mesh, while in multiple space dimensions, additional constraints are needed to specify mesh shapes and orientations. The meshes are automatically evolved with the underlying solution, typically by solving a moving mesh partial differential equation (MMPDE).

While being very general, the "conventional" moving mesh method has the following issues when applied to singularity detection. First, it requires explicit knowledge of the singularity, for example, its scaling exponent, in order to correctly capture the singularity [46]. Second, it tends to place too many mesh points near the singularity while leaving too few elsewhere, which can cause instability. Third, mesh smoothing, an operation necessary for maintaining stability, can significantly limit the maximum resolution power of the mesh. Finally, the moving mesh method computes only a discrete approximation of the mesh mapping function, which can result in catastrophic loss of accuracy in the computation of a singularity (see section 3.3).

For the particular blowup candidate considered in this paper, preliminary uniform mesh computations suggest that the vorticity function tends to concentrate at a single point. In addition, the solution appears to remain slowly varying and smooth outside a small neighborhood of the singularity. These observations motivate the following special mesh adaptation strategy.

The adaptive mesh covering the computational domain $D\left(1, \frac{1}{4} L\right)$ is constructed from a pair of analytic mesh mapping functions,

$$
r=r(\rho), \quad z=z(\eta),
$$

which are defined on $[0,1]$, are infinitely differentiable, and have a density that is even at both 0 and 1 . The even symmetries of the mesh density ensure that the resulting mesh can be extended smoothly to the full cylinder $D(1, L)$. The mesh mapping functions contain a small number of parameters, which are dynamically adjusted so that a certain fraction of the mesh points (e.g., $50 \%$ along each dimension) is placed in a small neighborhood of the singularity. Once the mesh mapping functions are constructed, the computational domain $D\left(1, \frac{1}{4} L\right)$ is covered with a tensor-product mesh:

$$
\mathcal{G}_{0}=\left\{\left(r_{j}, z_{i}\right): 0 \leq i \leq M, 0 \leq j \leq N\right\}
$$


where

$$
r_{j}=r\left(j h_{r}\right), z_{i}=z\left(i h_{z}\right), \quad h_{r}=1 / N, h_{z}=1 / M .
$$

The precise definition and construction of the mesh mapping functions can be found in [58, Appendix A].

The mesh is evolved using the following procedure. Starting from a reference time $t_{0}$, the "singularity region" $S_{0}$ at $t_{0}$ is identified as the smallest rectangle in the $r z$-plane that encloses the set

$$
D_{\delta_{0}}\left(t_{0}\right):=\left\{(r, z) \in D\left(1, \frac{1}{4} L\right):\left|\omega\left(r, z, t_{0}\right)\right| \geq \delta_{0}\left\|\omega\left(\cdot, t_{0}\right)\right\|_{\infty}\right\}, \quad \delta_{0} \in(0,1) .
$$

Once $S_{0}$ is determined, an adaptive mesh $\mathcal{G}_{0}$ is fit to $S_{0}$ and the solution is advanced in the $\rho \eta$-space by one time step to $t_{1}$. The singularity region $S_{1}$ at $t_{1}$ is then computed and compared with $S_{0}$. If the ratios between the sides of $S_{1}$ and $S_{0}$ (in either dimension) drop below a certain threshold (e.g., 80\%), which indicates the support of the maximum vorticity has shrunk by a sufficient amount, or if the maximum vorticity at $t_{1}$ is "too close" to the boundaries of $S_{0}$,

$$
\max _{(r, z) \in \partial S_{0}}\left|\omega\left(r, z, t_{1}\right)\right| \geq \delta_{1}\left\|\omega\left(\cdot, t_{1}\right)\right\|_{\infty}, \quad \delta_{1} \in\left(\delta_{0}, 1\right),
$$

which indicates the maximum vorticity is about to leave $S_{0}$, then a new mesh $\mathcal{G}_{1}$ is computed and adapted to $S_{1}$. In the event of a mesh update, the solution is interpolated from $\mathcal{G}_{0}$ to $\mathcal{G}_{1}$ in the $\rho \eta$-space using an 8th-order piecewise polynomial interpolation in $\rho$ and a spectral interpolation in $\eta$. The whole procedure is then repeated with $\mathcal{G}_{0}$ replaced by $\mathcal{G}_{1}$ and $t_{0}$ replaced by $t_{1}$.

We remark that the mesh update criterion (3.1) is designed to prevent the peak vorticity from escaping the singularity region, as is the case in one of our earlier computations where the singularity keeps moving toward the symmetry axis. Since in the current computation the singularity is fixed at the corner $\tilde{q}_{0}=(1,0)^{T}$, the criterion (3.1) has practically no effect.

The mesh adaptation strategy described above has several advantages compared with the conventional moving mesh method. First, it can automatically resolve a self-similar singularity regardless of its scalings, provided that the singularity has a bell-shaped similarity profile, which is what we observe in our case (see Figure 1(b)). This is crucial to the success of our computations, because the (axisymmetric) Euler equations allow for infinitely many self-similar scalings (see section 4.7), which means that the scaling exponent of the singularity cannot be determined a priori. Second, the method always places enough mesh points (roughly $50 \%$ along each dimension) outside the singularity region, ensuring a well-behaved and stable mesh (see section 4.1). Third, the explicit control of the mesh mapping functions eliminates the need of mesh smoothing, which allows the mesh to achieve arbitrarily high resolutions. Finally, the analytic representation of the mesh mapping functions ensures accurate approximations of space derivatives, hence greatly improving the quality of the computed solutions (see section 3.3).

3.2. The B-Spline-Based Galerkin Poisson Solver. A key observation we made from our computations is that the overall accuracy of the numerical solution of the initial-boundary value problem (2.1)-(2.2) depends crucially on the accuracy of the Poisson solver. Among the methods commonly used for solving Poisson equations, namely, finite difference, finite element Galerkin, and finite element collocation, we 
have chosen the Galerkin method both for its high accuracy and for its rigorous theoretic framework, which makes the error analysis much easier.

We have designed and implemented a B-spline-based Galerkin method for the Poisson equation (2.1c). Compared with the "conventional" Galerkin methods based on piecewise polynomials, the B-spline-based method requires no mesh generation and hence is much easier to implement. More importantly, the method can achieve arbitrary global smoothness and approximation order with relative ease and few degrees of freedom, in contrast to the conventional piecewise-polynomial-based methods. This makes the method a natural choice for our problem.

The Poisson equation (2.1c) is solved in the $\rho \eta$-space using the following procedure. First, the equation is recast in the $\rho \eta$-coordinates:

$$
-\frac{1}{r^{3} r_{\rho}}\left(r^{3} \frac{\psi_{\rho}}{r_{\rho}}\right)_{\rho}-\frac{1}{z_{\eta}}\left(\frac{\psi_{\eta}}{z_{\eta}}\right)_{\eta}=\omega, \quad(\rho, \eta) \in[0,1]^{2}
$$

where for clarity we have written $\psi$ for $\psi_{1}$ and $\omega$ for $\omega_{1}$. Next, the equation is multiplied by $r^{3} r_{\rho} z_{\eta} \phi$ and is integrated over the domain $[0,1]^{2}$, where $\phi \in V$ (to be defined below) is a suitable test function. After a routine integration by parts, this yields the desired weak formulation of (2.1c), which reads as follows: Find $\psi \in V$ such that

$$
\begin{aligned}
a(\psi, \phi) & :=\int_{[0,1]^{2}}\left(\frac{\psi_{\rho}}{r_{\rho}} \frac{\phi_{\rho}}{r_{\rho}}+\frac{\psi_{\eta}}{z_{\eta}} \frac{\phi_{\eta}}{z_{\eta}}\right) r^{3} r_{\rho} z_{\eta} d \rho d \eta \\
& =\int_{[0,1]^{2}} \omega \phi r^{3} r_{\rho} z_{\eta} d \rho d \eta=: f(\phi) \quad \forall \phi \in V,
\end{aligned}
$$

where (recall the odd symmetry of $\psi$ at $\eta=0,1$ )

$$
\begin{aligned}
V=\operatorname{span}\left\{\phi \in H^{1}[0,1]^{2}: \phi(-\rho, \eta)\right. & =\phi(\rho, \eta), \\
\phi(1, \eta) & =0, \phi(\rho, \ell-\eta)=-\phi(\rho, \ell+\eta) \forall \ell \in \mathbb{Z}\} .
\end{aligned}
$$

To introduce Galerkin approximation, we define the finite-dimensional subspace of weighted uniform B-splines [41] of even order $k$ :

$$
V_{h}:=V_{w, h}^{k}=\operatorname{span}\left\{w(\rho) b_{j, h_{r}}^{k}(\rho) b_{i, h_{z}}^{k}(\eta)\right\} \cap V,
$$

where $w(\rho)$ is a nonnegative weight function of order 1 vanishing on $\rho=1$,

$$
w(\rho) \sim(1-\rho), \quad \rho \rightarrow 1^{-},
$$

and $b_{\ell, h}^{k}(s)=b^{k}((s / h)-(\ell-k / 2))$ are shifted and rescaled uniform B-splines of order $k$. The Galerkin formulation then reads as follows: Find $\psi_{h} \in V_{h}$ such that

$$
a\left(\psi_{h}, \phi_{h}\right)=f\left(\phi_{h}\right) \quad \forall \phi_{h} \in V_{h} .
$$

With suitably chosen basis functions of $V_{h}$, this gives rise to a symmetric, positive definite linear system $A x=b$ which can be solved to yield the Galerkin solution $\psi_{h}$. A detailed construction of the linear system can be found in [58, Appendix B].

The parameters used in our computations are $k=6$ and $w(\rho)=1-\rho^{2}$. 
Using the theory of quasi-interpolants [41], it can be shown that

$$
\int_{[0,1]^{2}}\left|\nabla \psi-\nabla \psi_{h}\right|^{2} r^{3} d r d z \leq C_{0} C_{r z}\left(h_{r} h_{z}\right)^{k-1} \int_{[0,1]^{2}} \sum_{|\alpha| \leq k-1}\left|\tilde{\partial}^{\alpha} \nabla \psi\right|^{2} r^{3} d r d z
$$

where $\nabla=\left(\partial_{r}, \partial_{z}\right)^{T}, \tilde{\partial}^{\alpha}=\partial_{\rho}^{\alpha_{1}} \partial_{\eta}^{\alpha_{2}}$ are differential operators in $r z$ - and $\rho \eta$-planes, respectively, $C_{r z}$ is a mesh mapping dependent constant, and $C_{0}$ is an absolute constant. In our computations, the constant $C_{r z}$ is observed to be very close to 1 for all times, which confirms the stability of the Galerkin solver.

3.3. The Overall Algorithm. Given an adaptive mesh $\mathcal{G}_{0}$ and the data $\left(u_{1}, \omega_{1}\right)$ defined on $\mathcal{G}_{0}$, the solution is advanced using the following procedure. First, the Poisson equation (2.1c) is solved for $\psi_{1}$ in the $\rho \eta$-space using a 6th-order B-spline-based Galerkin method (section 3.2). Second, the $2 \mathrm{D}$ velocity $\tilde{u}=\left(u^{r}, u^{z}\right)^{T}$ is evaluated at the grid points using $(2.1 \mathrm{~d})$. Third, an adaptive time step $\delta_{t}$ is computed on $\mathcal{G}_{0}$ so that the CFL condition is satisfied with a suitably small CFL number $\nu$ (e.g., 0.5), and the relative growth of the solution in one step does not exceed a small threshold $\epsilon_{t}$ (e.g., $\left.5 \%\right)$. Finally, the solution $\left(u_{1}, \omega_{1}\right)$ is advanced according to $(2.1 \mathrm{a})-(2.1 \mathrm{~b})$ by $\delta_{t}$ using an explicit 4th-order Runge-Kutta method, and the mesh $\mathcal{G}_{0}$ is adapted to the new solution if necessary (section 3.1).

In the last step of the algorithm, the evolution equations for $u_{1}$ and $\omega_{1}$ are semidiscretized in the $\rho \eta$-space, where the space derivatives are expressed in the $\rho \eta$-coordinates and are approximated using 6th-order centered difference formulas, e.g.,

$$
v_{r}\left(r_{j}, z_{i}\right)=:\left(v_{r}\right)_{i j}=\frac{\left(v_{\rho}\right)_{i j}}{\left(r_{\rho}\right)_{j}} \approx \frac{1}{\left(r_{\rho}\right)_{j}}\left(Q_{\rho, 6} v_{i, .}\right)_{j}, \quad v=u_{1} \text { or } \omega_{1} .
$$

Here, as usual,

$$
Q_{\rho, 6}:=D_{\rho, 0}\left(I-\frac{1}{6} h_{r}^{2} D_{\rho,+} D_{\rho,-}+\frac{1}{30} h_{r}^{4} D_{\rho,+}^{2} D_{\rho,-}^{2}\right)
$$

denotes the standard 6th-order centered approximation to $\partial_{\rho}$, and

$$
\left(D_{\rho, \pm} v_{i,}\right)_{j}:= \pm \frac{1}{h_{r}}\left(v_{i, j \pm 1}-v_{i, j}\right), \quad\left(D_{\rho, 0} v_{i, .}\right)_{j}:=\frac{1}{2 h_{r}}\left(v_{i, j+1}-v_{i, j-1}\right)
$$

denote the standard forward, backward, and centered difference operators, respectively. Note that the derivative $r_{\rho}$ of the mesh mapping function is computed directly from the analytic representation of $r$ without any difference approximation. This is crucial for the accurate evaluation of $v_{r}$, especially in "singularity regions" where the inverse mesh density $r_{\rho}$ is close to 0 and is nearly constant [58, Appendix A]. When $r_{\rho}$ is small and nearly constant, a high-order difference approximation of $r_{\rho}$ tends to be contaminated by catastrophic cancellation, and the discretely approximated values of $r_{\rho}$ can have large relative errors or even become negative, causing failures of the adaptive mesh algorithm and hence the entire computation. By computing $r_{\rho}$ directly from the analytic representation of $r$, this problem is avoided and the solution is ensured to be accurately approximated even in regions where the singularity is about to form and where $r_{\rho} \approx c \ll 1$. This also explains why the conventional moving mesh method is not suitable for singularity computations where high accuracy is demanded, because the method computes only a discrete approximation of the mesh mapping function, which necessarily requires a difference approximation of $r_{\rho}$ in the evaluation of a space 
derivative $v_{r}$. Without mesh smoothing, this can cause instability, while with mesh smoothing the mesh resolution will inevitably be limited, which is undesired.

The centered difference formulas described above need to be supplemented by numerical boundary conditions near $\rho, \eta=0,1$. Along the $\eta$-dimension, the symmetry condition

$$
v_{-i, j}=-v_{i, j}, \quad v_{M+i, j}= \pm v_{M-i, j}, \quad 1 \leq i \leq 3,0 \leq j \leq N,
$$

is used near $\eta=0$ and $\eta=1$, where the + sign applies to $u_{1}$ and the - sign applies to $\omega_{1}$. Along the $\rho$-dimension, the symmetry condition

$$
v_{i,-j}=v_{i, j}, \quad 0 \leq i \leq M, 1 \leq j \leq 3,
$$

is used near the axis $\rho=0$ and the extrapolation condition

$$
\left(D_{\rho,-}^{7} v_{i,}\right)_{N+j}=0, \quad 0 \leq i \leq M, 1 \leq j \leq 3,
$$

is applied near the solid boundary $\rho=1 .^{5}$ The extrapolation condition is known to be GKS (Gustafsson-Kreiss-Sundstrom) stable for linear hyperbolic problems [39, Theorem 13.1.3], and it is expected to remain stable when applied to the Euler equations as long as the underlying solution is sufficiently smooth.

4. Numerical Results. The initial-boundary value problem $(2.1)-(2.2)$ is numerically solved on the quarter cylinder $D\left(1, \frac{1}{24}\right)$ (with $L=\frac{1}{6}$ ), and the results strongly suggest the formation of a finite-time singularity in the computed solutions. The purpose of this section is to provide, through careful analysis, convincing evidence to support the existence of such a singularity. The presentation begins with an overview of the computations in sections 4.1-4.2, where the effectiveness of the adaptive mesh is demonstrated and the first sign of a finite-time singularity is displayed. After a careful resolution study of the computed solutions in section 4.3, we proceed to sections 4.4-4.5, where the asymptotic scalings of the vorticity moments are analyzed and the divergence of the time integral of the maximum vorticity, and hence the blowup of the computed solutions, is confirmed. This conclusion is further strengthened in section 4.6, where the geometric structures of the vorticity direction field are investigated and the consistency between the blowing-up solutions and the various geometric nonblowup criteria is demonstrated. Once the existence of a finite-time singularity is confirmed, we move on to sections $4.7-4.8$, where the locally self-similar structure of the blowing-up solutions is examined and a physical interpretation of the finitetime singularity is attempted. The discussion is concluded in section 4.9 , where a $1 \mathrm{D}$ model featuring some of the essential properties of the blowup is investigated, and where existence of finite-time singularities for this 1D model is rigorously established.

4.I. Effectiveness of the Adaptive Mesh. We have numerically solved the problem (2.1)-(2.2) on meshes of size $256 k \times 256 k$, where $k=4,5,6,7,8$. In each computation, the solution is initialized on a uniform mesh, which is then adjusted to the initial condition using the adaptive mesh algorithm described in section 3.1. Once an "optimal" mesh is obtained, the solution is advanced indefinitely in time using the method described in section 3 , until either the time step drops below $10^{-12}$, or the minimum mesh spacing drops below $\epsilon_{r}=10^{-15}$ (in $r$ ) or $\epsilon_{z}=10^{-15}\left(\frac{1}{4} L\right)$ (in $z$ ), whichever happens first.

\footnotetext{
${ }^{5}$ While a 6th-order extrapolation condition $\left(D_{\rho,-}^{6} v_{i, .}\right)_{N+j}=0$ suffices to maintain a formal 6thorder accuracy for the overall scheme, we choose the higher-order extrapolation condition for better accuracy.
} 
Table I Stopping time $t_{e}$ and cause of termination, where $\left(\delta_{r}, \delta_{z}\right)$ denote the minimum mesh spacing in $r$ and $z$, respectively.

\begin{tabular}{ccc}
\hline Mesh size & $t_{e}$ & Cause of termination \\
\hline $1024 \times 1024$ & 0.0035055667206 & $\delta_{r}<\epsilon_{r}$ and $\delta_{z}<\epsilon_{z}$ \\
$1280 \times 1280$ & 0.0035055581996 & $\delta_{z}<\epsilon_{z}$ \\
$1536 \times 1536$ & 0.0035055522856 & $\delta_{z}<\epsilon_{z}$ \\
$1792 \times 1792$ & 0.0035055523092 & $\delta_{r}<\epsilon_{r}$ and $\delta_{z}<\epsilon_{z}$ \\
$2048 \times 2048$ & 0.0035055472037 & $\delta_{r}<\epsilon_{r}$ and $\delta_{z}<\epsilon_{z}$ \\
\hline
\end{tabular}

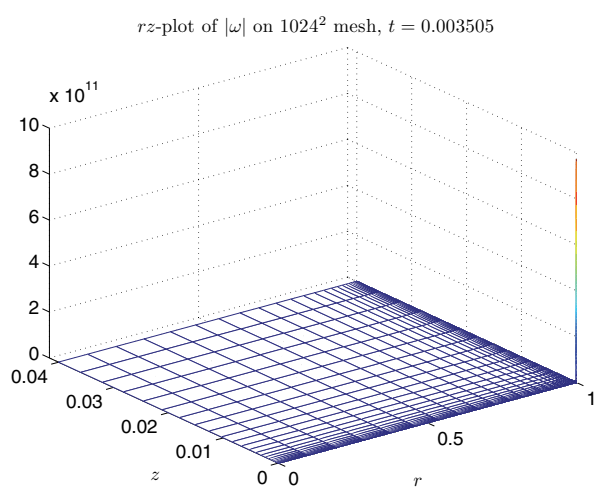

(a) $r z$-plane

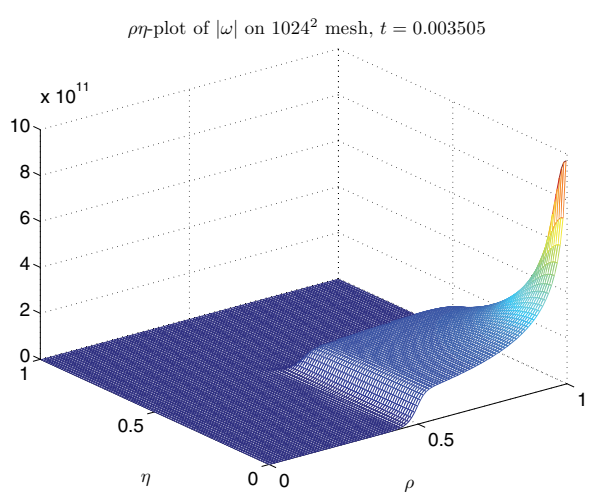

(b) $\rho \eta$-plane

Fig. I The vorticity function $|\omega|$ on the $1024 \times 1024$ mesh at $t=0.003505$, in (a) rz-coordinates and (b) $\rho \eta$-coordinates, where for clarity only one-tenth of the mesh lines are displayed along each dimension.

Table 1 shows the stopping time $t_{e}$ and the cause of termination for each resolution run. For all the computations shown in the table, the total number of remeshing steps is about 130, and this number depends on the choice of the parameters $\delta_{0}, \delta_{1}$ (cf. (3.1)) but is relatively insensitive to the particular choice of the mesh mapping functions. As indicated by the mostly decreasing stopping time (with respect to the increasing resolution) and the vanishing minimum mesh spacings, the solution seems to develop a very singular structure in finite time. To determine the nature of the singular structure and to see how well the adaptive mesh resolves it, we plot in Figure 1 the vorticity function $|\omega|$ computed on the $1024 \times 1024$ mesh at $t=0.003505$, in both the $r z$-coordinates (Figure 1(a)) and the $\rho \eta$-coordinates (Figure 1(b)). The $r z$ plot suggests that the singular structure could be a point-singularity at the corner $\tilde{q}_{0}=(1,0)^{T}$, which corresponds to a ring-singularity on the solid boundary due to the axial symmetry. The $\rho \eta$-plot, on the other hand, shows that a good portion of the mesh points (roughly 50\% along each dimension) are consistently placed in regions where $|\omega|$ is comparable with the maximum vorticity $\|\omega\|_{\infty}$, hence demonstrating the effectiveness of the adaptive mesh. To quantitatively measure the maximum resolution power achieved by the adaptive mesh, we define the mesh compression ratios

$$
p_{\infty}:=\frac{L}{4 z^{\prime}\left(\eta_{\infty}\right)}, \quad q_{\infty}:=\frac{1}{r^{\prime}\left(\rho_{\infty}\right)}
$$


Table 2 Mesh compression ratios $\left(p_{\infty}, q_{\infty}\right)$ and effective mesh resolutions $\left(M_{\infty}, N_{\infty}\right)$ at the location of the maximum vorticity at $t=0.003505$.

\begin{tabular}{ccccc}
\hline \multirow{2}{*}{ Mesh size } & \multicolumn{4}{c}{$t=0.003505$} \\
\cline { 2 - 5 } & $p_{\infty}$ & $M_{\infty}$ & $q_{\infty}$ & $N_{\infty}$ \\
\hline $1024 \times 1024$ & $1.9456 \times 10^{9}$ & $1.9923 \times 10^{12}$ & $1.6316 \times 10^{9}$ & $1.6708 \times 10^{12}$ \\
$1280 \times 1280$ & $1.9530 \times 10^{9}$ & $2.4999 \times 10^{12}$ & $1.6285 \times 10^{9}$ & $2.0844 \times 10^{12}$ \\
$1536 \times 1536$ & $1.9444 \times 10^{9}$ & $2.9866 \times 10^{12}$ & $1.6328 \times 10^{9}$ & $2.5079 \times 10^{12}$ \\
$1792 \times 1792$ & $1.9504 \times 10^{9}$ & $3.4951 \times 10^{12}$ & $1.6344 \times 10^{9}$ & $2.9288 \times 10^{12}$ \\
$2048 \times 2048$ & $1.9503 \times 10^{9}$ & $3.9942 \times 10^{12}$ & $1.6330 \times 10^{9}$ & $3.3444 \times 10^{12}$ \\
\hline
\end{tabular}

Table 3 Time step $\delta_{t}$ at selected time $t$.

\begin{tabular}{cccccc}
\hline \multirow{2}{*}{ Mesh size } & \multicolumn{5}{c}{$\delta_{t}$} \\
\cline { 2 - 6 } & $t=0^{\dagger}$ & $t=0.003$ & $t=0.0034$ & $t=0.0035$ & $t=0.003505$ \\
\hline $1024 \times 1024$ & $1 \times 10^{-6}$ & $2.8754 \times 10^{-7}$ & $4.9502 \times 10^{-8}$ & $2.8831 \times 10^{-9}$ & $2.4240 \times 10^{-10}$ \\
$1280 \times 1280$ & $1 \times 10^{-6}$ & $2.3120 \times 10^{-7}$ & $3.9636 \times 10^{-8}$ & $2.2983 \times 10^{-9}$ & $2.5772 \times 10^{-10}$ \\
$1536 \times 1536$ & $1 \times 10^{-6}$ & $1.9165 \times 10^{-7}$ & $3.2907 \times 10^{-8}$ & $1.9165 \times 10^{-9}$ & $2.2223 \times 10^{-10}$ \\
$1792 \times 1792$ & $1 \times 10^{-6}$ & $1.6578 \times 10^{-7}$ & $2.8451 \times 10^{-8}$ & $1.6418 \times 10^{-9}$ & $1.9122 \times 10^{-10}$ \\
$2048 \times 2048$ & $1 \times 10^{-6}$ & $1.4509 \times 10^{-7}$ & $2.4046 \times 10^{-8}$ & $1.4367 \times 10^{-9}$ & $2.0272 \times 10^{-10}$ \\
\hline
\end{tabular}

${ }^{\dagger}$ The maximum time step allowed in our computations is $10^{-6}$.

Table 4 Maximum vorticity $\|\omega\|_{\infty}$ at selected time $t$.

\begin{tabular}{cccccc}
\hline \multirow{2}{*}{ Mesh size } & \multicolumn{5}{c}{$\|\omega\|_{\infty}$} \\
\cline { 2 - 6 } & $t=0$ & $t=0.003$ & $t=0.0034$ & $t=0.0035$ & $t=0.003505$ \\
\hline $1024 \times 1024$ & $3.7699 \times 10^{3}$ & $9.0847 \times 10^{4}$ & $4.3127 \times 10^{6}$ & $5.8438 \times 10^{9}$ & $1.2416 \times 10^{12}$ \\
$1280 \times 1280$ & $3.7699 \times 10^{3}$ & $9.0847 \times 10^{4}$ & $4.3127 \times 10^{6}$ & $5.8423 \times 10^{9}$ & $1.2407 \times 10^{12}$ \\
$1536 \times 1536$ & $3.7699 \times 10^{3}$ & $9.0847 \times 10^{4}$ & $4.3127 \times 10^{6}$ & $5.8417 \times 10^{9}$ & $1.2403 \times 10^{12}$ \\
$1792 \times 1792$ & $3.7699 \times 10^{3}$ & $9.0847 \times 10^{4}$ & $4.3127 \times 10^{6}$ & $5.8415 \times 10^{9}$ & $1.2401 \times 10^{12}$ \\
$2048 \times 2048$ & $3.7699 \times 10^{3}$ & $9.0847 \times 10^{4}$ & $4.3127 \times 10^{6}$ & $5.8413 \times 10^{9}$ & $1.2401 \times 10^{12}$ \\
\hline
\end{tabular}

and the effective mesh resolutions

$$
M_{\infty}:=p_{\infty} M=\frac{L M}{4 z^{\prime}\left(\eta_{\infty}\right)}, \quad N_{\infty}:=q_{\infty} N=\frac{N}{r^{\prime}\left(\rho_{\infty}\right)}
$$

at the location $\left(\rho_{\infty}, \eta_{\infty}\right)^{T} \equiv(1,0)^{T}$ of the maximum vorticity $\|\omega\|_{\infty}$. The values of these quantities computed at $t=0.003505$ are summarized in Table 2, from which the power of the adaptive mesh can be clearly observed.

The above analysis confirms the effectiveness of the adaptive mesh in the "inner region" where the vorticity function $|\omega|$ is most singular. The quality of the mesh outside the inner region, as well as the stability and convergence of the Poisson solver (cf. section 3.2) on the highly nonuniform adaptive mesh, can be ensured from a separate analysis. Interested readers are referred to [58, section 4.1] for details.

4.2. First Sign of Singularity. To examine more closely the nature of the singular structure observed in Figure 1, we report in Tables 3-4 the (variable) time steps $\delta_{t}$ and the maximum vorticity $\|\omega\|_{\infty}$ recorded at selected time instants $t$. We also plot in Figure 2 the double logarithm of the maximum vorticity, $\log \left(\log \|\omega\|_{\infty}\right)$, computed on the coarsest $1024 \times 1024$ and the finest $2048 \times 2048$ meshes. It can be observed from 


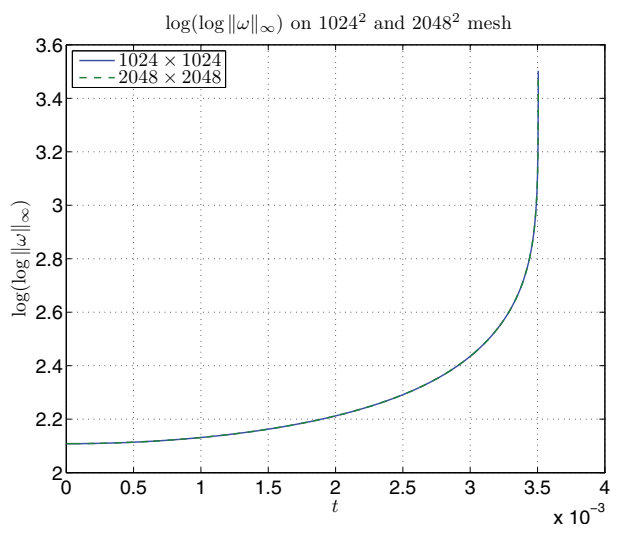

Fig. 2 The double logarithm of the maximum vorticity $\log \left(\log \|\omega\|_{\infty}\right)$ computed on the $1024 \times 1024$ and the $2048 \times 2048$ meshes. The two curves overlap and are virtually indistinguishable from each other (see section 4.3 below for a detailed resolution study on the nearly singular solutions).

these results that, for each computation, there exists a short time interval right before the stopping time $t_{e}$ in which the solution grows tremendously. This can be readily inferred from the sharp decrease in time step $\delta_{t}$ (Table 3 ) as well as the super-doubleexponential growth of the maximum vorticity $\|\omega\|_{\infty}$ (Table 4, Figure 2). In addition, the nearly singular solution seems to converge under mesh refinement (Table 4). These behaviors are characteristic of a blowing-up solution and may be viewed as the first sign of a finite-time singularity looming on the horizon.

4.3. Resolution Study. Of course, neither a rapidly decreasing time step nor a fast growing vorticity can be used alone as evidence for a finite-time singularity. To investigate the issue of finite-time blowup more closely, a much more thorough analysis is needed which, in the first place, requires a careful examination of the accuracy of the computed solutions.

There are several well-established "standard" methods in the literature to gauge the quality of an Euler computation:

(i) Energy conservation. It is well known that, under suitable regularity assumptions, the solutions of the Euler equations conserve the kinetic energy

$$
E=\frac{1}{2} \int_{D(1, L)}|u|^{2} d x=\frac{1}{2} \int_{0}^{1} \int_{0}^{L}\left(\left|u_{1}\right|^{2}+\left|\psi_{1, r}\right|^{2}+\left|\psi_{1, z}\right|^{2}\right) r^{3} d r d z .
$$

Thus a widely used "quality indicator" for Euler computations is the relative change of the energy integral $E$ over time.

(ii) Enstrophy and enstrophy production rate. Another widely accepted "error indicator" for Euler computations is the enstrophy integral

$$
\mathcal{E}=\int_{D(1, L)}|\nabla u|^{2} d x=\int_{D(1, L)}|\omega|^{2} d x
$$

and the enstrophy production rate integral

$$
\mathcal{E}_{p}:=\frac{d \mathcal{E}}{d t}=2 \int_{D(1, L)} \omega \cdot S \omega d x, \quad S=\frac{1}{2}\left(\nabla u+\nabla u^{T}\right) .
$$


These quantities are not conserved over time, but their convergence under mesh refinement provides partial evidence on the convergence of the underlying numerical solutions.

(iii) Energy spectra. For problems defined on periodic domains, it is also a common practice to perform convergence analysis on the energy spectra of the periodic velocity field $u$,

$$
E_{p}(k)=\sum_{|\ell| \in(k-1 / 2, k+1 / 2]}\left|\hat{u}_{\ell}\right|^{2},
$$

and use the results as a measure of the quality of the underlying solutions. Here, as usual, $\hat{u}_{\ell}$ denotes the vector Fourier coefficients of the velocity $u$, which on an $L_{1} \times L_{2} \times L_{3}$ periodic box $B$ is defined by

$$
\begin{aligned}
\hat{u}_{\ell} & =\frac{1}{|B|} \int_{B} u \mathrm{e}^{-\mathrm{i} \ell \cdot x} d x \\
& =\frac{1}{L_{1} L_{2} L_{3}} \int_{0}^{L_{3}} \mathrm{e}^{-\mathrm{i} \ell_{3} x_{3}} \int_{0}^{L_{2}} \mathrm{e}^{-\mathrm{i} \ell_{2} x_{2}} \int_{0}^{L_{1}} u \mathrm{e}^{-\mathrm{i} \ell_{1} x_{1}} d x_{1} d x_{2} d x_{3} .
\end{aligned}
$$

(iv) Maximum vorticity. Perhaps one of the most important quantities in the regularity theory of the Euler equations, the maximum vorticity

$$
\|\omega\|_{\infty}:=\|\omega\|_{L^{\infty}(D(1, L))}=\max _{(r, z) \in D(1, L)}|\omega(r, z)|
$$

is closely monitored in most Euler computations, and its convergence under mesh refinement is also frequently used as a "quality indicator" for the underlying numerical simulations.

(v) Conservation of circulation. In a more recent work [10], the relative change of the circulation

$$
\Gamma=\oint_{C} u \cdot d s
$$

around selected material curves $C$ is proposed as an "error indicator" for Euler computations. The idea is that, according to Kelvin's circulation theorem, the circulation around any closed material curve $C$ is conserved by an Euler flow, and hence the same should be expected for a numerical solution as well. While conservation of circulation is a physically important principle, its numerical confirmation is not always plausible, because it is not always clear how to choose the "representative" material curves $C$. In addition, it is generally not easy to follow a material curve in an Euler flow, since most such simulations are performed on Eulerian meshes, while tracking a material curve requires the use of a Lagrangian mesh.

We argue that none of the above "quality indicators" is adequate for the purpose of singularity detection. Admittedly, energy, enstrophy, and circulation are all physically significant quantities, and without a doubt they should all be accurately resolved in any "reasonable" Euler simulations. On the other hand, it is also important to realize that these quantities are global quantities and do not measure the accuracy of a numerical solution at any particular point or even in any particular subset of the computational domain. Since blowing-up solutions of the Euler equations must be characterized by rapidly growing vorticity [4], and in most cases such intense vorticity amplification is realized in spatial regions with rapidly collapsing support [50, 44], it is crucial that the accuracy of a numerically detected blowup candidate be measured by local error metrics such as the pointwise (sup-norm) error. When restricted to 
bounded domains, the pointwise error is stronger than any other global error metrics in the sense that the latter can be easily bounded in terms of the former, while the converse does not hold true in general. Consequently, the pointwise error provides the most stringent measure for the quality of a blowup candidate, both near the point of blowup and over the entire (bounded) computational domain.

Arguing in a similar manner, we see that neither energy spectrum nor maximum vorticity gives an adequate measure of error for a potentially blowing-up solution. On the one hand, the construction of an energy spectrum removes the phase information and reduces the dimension of the data from three to one, leaving only an incomplete picture of a solution and hence of its associated error. On the other hand, maximum vorticity, albeit significant in its own right, does not tell us anything about a solution except at the point where the vorticity magnitude attained its maximum.

In view of the above considerations, we shall gauge the quality of our Euler simulations at any fixed time instant $t$ using the sup-norm relative errors of the computed solutions $\left(u_{1}, \omega_{1}, \psi_{1}\right)$. More specifically, we shall estimate the error of a given solution, say $u_{1}$, by comparing it with a "reference solution," say $\hat{u}_{1}$, that is computed at the same time $t$ on a finer mesh. The reference solution $\hat{u}_{1}$ is first interpolated to the coarse mesh on which $u_{1}$ is defined. Then the maximum difference between the two solutions is computed and the result is divided by the maximum of $\left|\hat{u}_{1}\right|$ (measured on the finer mesh) to yield the desired relative error.

We check the accuracy of our computations in five steps.

4.3. I. Code Validation on Test Problems. First, we apply the numerical method described in section 3 to a test problem with known exact solutions and artificially generated external forcing terms [58, Appendix C]. The exact solutions are chosen to mimic the behavior of the blowing-up Euler solution computed from (2.1)-(2.2), and numerical experiments on successively refined meshes confirm the 6th-order convergence of the overall method [58, Table 7].

4.3.2. Resolution Study on Transformed Primitive Variables. Second, we perform a resolution study on the actual solutions of problem (2.1)-(2.2) at various time instants $t$, up to the time $t=0.003505$ shortly before the simulations terminate. For each $256 k \times 256 k$ mesh except for the finest one, we compare the solution $\left(u_{1}, \omega_{1}, \psi_{1}\right)$ computed on this mesh with the reference solution $\left(\hat{u}_{1}, \hat{\omega}_{1}, \hat{\psi}_{1}\right)$ computed at the same time $t$ on the finer $[256(k+1)] \times[256(k+1)]$ mesh, and compute the sup-norm relative error using the procedure described above. For each $256 k \times 256 k$ mesh except for the coarsest one, we also compute, for each error $e_{k}$ defined on this mesh, the numerical order of convergence

$$
\beta_{k}=\log _{k /(k-1)}\left(\frac{e_{k-1}}{e_{k}}\right) .
$$

Here, the error $e_{k}$ is understood as a function of the (uniform) mesh spacing $h_{r}=$ $h_{z}=1 /(256 k)$ in the $\rho \eta$-space, and it is assumed to admit an asymptotic expansion in powers of $h_{r}$ and $h_{z}$. Under suitable regularity assumptions on the underlying exact solutions and with suitable choices of time steps, it can be shown that $\beta_{k}$ converges to its theoretical value ( 6 in this case) as $k \rightarrow \infty$.

The results of the resolution study on the primitive variables $\left(u_{1}, \omega_{1}, \psi_{1}\right)$ among the five mesh resolutions are summarized in Figure 3. To examine more closely the errors at the times when the solutions are about to "blow up," we also report in Table 5 the estimated sup-norm errors and numerical orders at $t=0.003505$. It can be observed from these results that, for small $t$, specifically for $t \lesssim 0.0015$, the solutions 


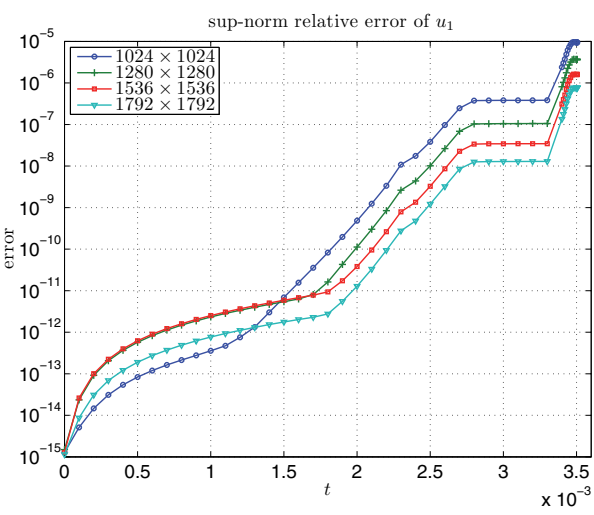

(a) sup-norm relative error of $u_{1}$

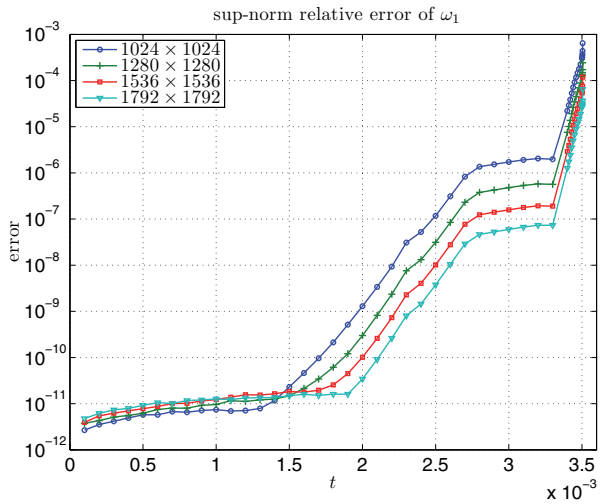

(c) sup-norm relative error of $\omega_{1}$

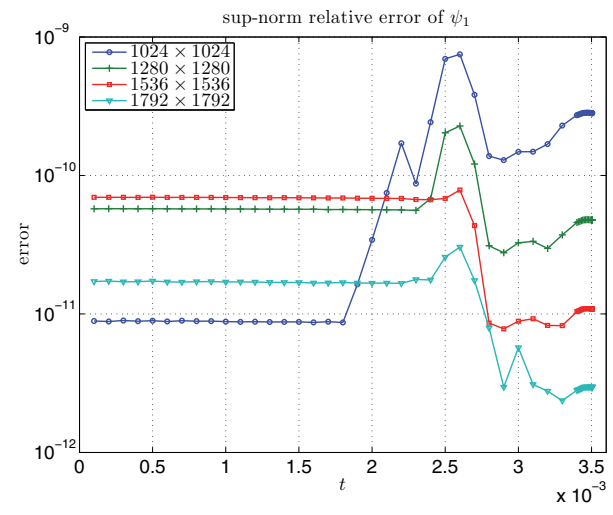

(e) sup-norm relative error of $\psi_{1}$

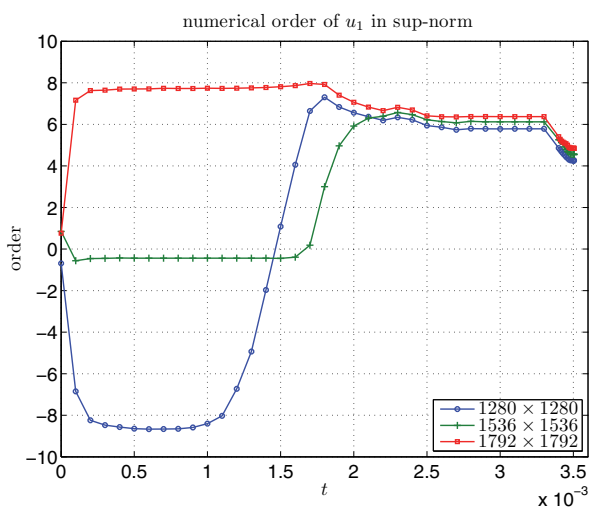

(b) numerical order of $u_{1}$ in sup-norm

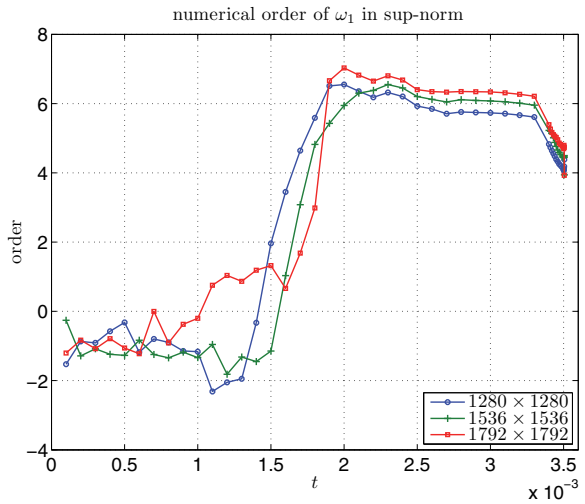

(d) numerical order of $\omega_{1}$ in sup-norm

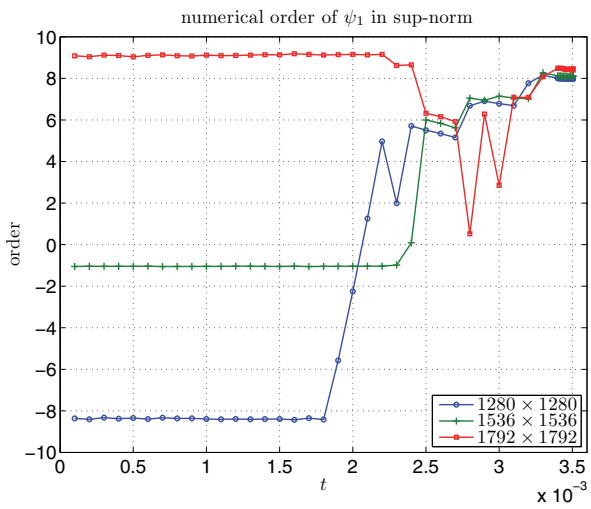

(f) numerical order of $\psi_{1}$ in sup-norm

Fig. 3 Resolution study in space: (a), (c), (e) sup-norm relative error and (b), (d), (f) numerical order in sup-norm of the transformed primitive variables $\left(u_{1}, \omega_{1}, \psi_{1}\right)$. The last time instant shown in the figure is $t=0.003505$.

are well resolved even on the coarsest $1024 \times 1024$ mesh, and further increase in mesh size does not lead to further improvement of the sup-norm errors. For $0.0015 \lesssim t \lesssim$ 0.0033 , the errors first grow exponentially in time and then level off after $t \approx 0.0028$. The numerical orders estimated on this time interval roughly match their theoretical 
Table 5 Sup-norm relative error and numerical order of convergence of the transformed primitive variables $\left(u_{1}, \omega_{1}, \psi_{1}\right)$ at $t=0.003505$. The absolute size of each variable, measured on the finest $2048 \times 2048$ mesh, is indicated in the last row of the table, "Sup-norm."

\begin{tabular}{ccccccc}
\hline \multirow{2}{*}{ Mesh size } & \multicolumn{7}{c}{ Sup-norm relative error at $t=0.003505$} \\
\cline { 2 - 7 } & $u_{1}$ & Order & $\omega_{1}$ & Order & $\psi_{1}$ & Order \\
\hline $1024 \times 1024$ & $9.4615 \times 10^{-6}$ & - & $6.4354 \times 10^{-4}$ & - & $2.8180 \times 10^{-10}$ & - \\
$1280 \times 1280$ & $3.6556 \times 10^{-6}$ & 4.26 & $2.4201 \times 10^{-4}$ & 4.38 & $4.7546 \times 10^{-11}$ & 7.97 \\
$1536 \times 1536$ & $1.5939 \times 10^{-6}$ & 4.55 & $1.1800 \times 10^{-4}$ & 3.94 & $1.0873 \times 10^{-11}$ & 8.09 \\
$1792 \times 1792$ & $7.5561 \times 10^{-7}$ & 4.84 & $6.4388 \times 10^{-5}$ & 3.93 & $2.9518 \times 10^{-12}$ & 8.46 \\
\hline Sup-norm & $1.0000 \times 10^{2}$ & - & $1.0877 \times 10^{6}$ & - & $2.1610 \times 10^{-1}$ & - \\
\hline
\end{tabular}

values 6 , confirming the full-order convergence of the computed solutions. For $t \gtrsim$ 0.0033 , the exponential growth of the sup-norm errors resumes at an accelerated pace, in correspondence with the strong, nonlinear amplifications of the underlying solutions observed in this stage. The numerical orders estimated for $u_{1}$ and $\omega_{1}$ decline slightly from 6 to 4 , as a result of the rapidly growing discretization error in time (Figure 5), while the ones for $\psi_{1}$ increase slightly from 6 to 8 , thanks most likely to the superconvergence property of the B-spline-based Poisson solver at grid points (section 3.2). Based on these observations, we conclude that the primitive variables computed on the finest two meshes have at least four significant digits up to and including the time $t=0.003505$ shortly before the singularity forms. To the best of our knowledge, this level of accuracy has never been observed in previous numerical studies (see also Table 11).

4.3.3. Resolution Study on Vorticity Vector. Since the Beale-Kato-Majda criterion suggests that the vorticity vector $\omega$ controls the blowup of smooth Euler solutions, we next perform a resolution study on $\omega$ to see how well it is resolved in our computations. The procedure is almost identical to that described for the primitive variables $\left(u_{1}, \omega_{1}, \psi_{1}\right)$, except that the difference between a vorticity vector $\omega$ and its reference value $\hat{\omega}$ needs to be measured in a suitable vector norm. By choosing the usual Euclidean norm, we have

$$
\begin{aligned}
|\omega-\hat{\omega}| & =\left\{\left(\omega^{r}-\hat{\omega}^{r}\right)^{2}+\left(\omega^{\theta}-\hat{\omega}^{\theta}\right)^{2}+\left(\omega^{z}-\hat{\omega}^{z}\right)^{2}\right\}^{1 / 2} \\
& =\left\{\left(r u_{1, z}-r \hat{u}_{1, z}\right)^{2}+\left(r \omega_{1, z}-r \hat{\omega}_{1, z}\right)^{2}+\left(2 u_{1}+r u_{1, r}-2 \hat{u}_{1}-r \hat{u}_{1, r}\right)^{2}\right\}^{1 / 2} .
\end{aligned}
$$

The resulting sup-norm errors and numerical orders are summarized in Figure 4 and Table 6 . These results will be used below in section 4.4 in the computation of the asymptotic scalings of the nearly singular solutions.

4.3.4. Resolution Study on Global Quantities. The next step in our resolution study is to examine the "conventional" error indicators defined using global quantities such as energy $E$, enstrophy $\mathcal{E}$, enstrophy production rate $\mathcal{E}_{p},{ }^{6}$ maximum vorticity $\|\omega\|_{\infty},{ }^{7}$ and circulation $\Gamma$. As we already pointed out, conservation of circulation is

\footnotetext{
${ }^{6}$ All these integrals are discretized in the $\rho \eta$-space using the 6th-order composite Boole's rule.

${ }^{7}$ We define $\|\omega\|_{\infty}$ simply as the maximum value of $|\omega|$ on the discrete mesh points (i.e., no interpolation is used to find the "precise" maximum). In view of the highly effective adaptive mesh, this does not cause any loss of accuracy. In addition, for the specific initial condition $(2.2 \mathrm{a}),\|\omega\|_{\infty}$ is always attained at $\tilde{q}_{0}=(1,0)^{T}$, which is always a mesh point.
} 


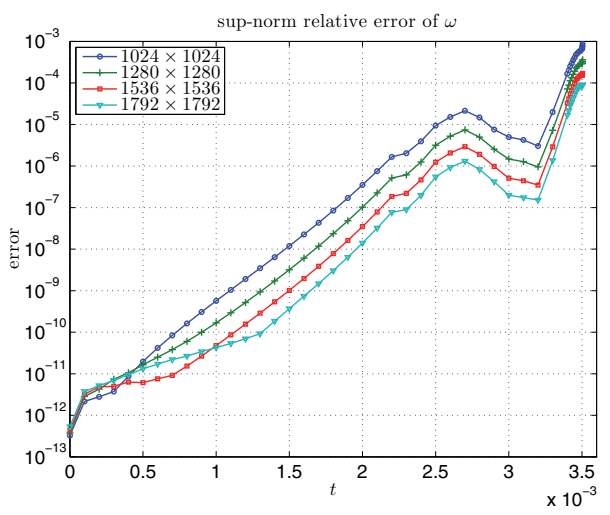

(a) sup-norm relative error

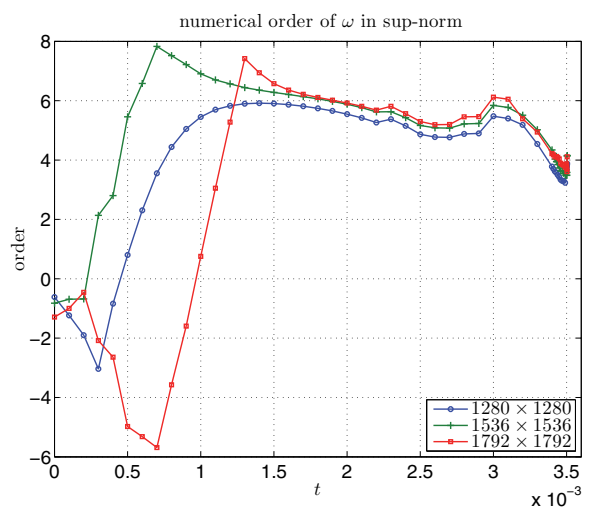

(b) numerical order in sup-norm

Fig. 4 Resolution study in space: (a) sup-norm relative error and (b) numerical order in sup-norm of the vorticity vector $\omega$. The last time instant shown in the figure is $t=0.003504$.

Table 6 Sup-norm relative error and numerical order of convergence of the vorticity vector $\omega$ at selected time $t$. The absolute size of $\omega$, measured on the finest $2048 \times 2048$ mesh, is indicated in the last row of the table, "Sup-norm."

\begin{tabular}{ccccc}
\hline \multirow{2}{*}{ Mesh size } & \multicolumn{5}{c}{ Sup-norm relative error of $\omega$} \\
\cline { 2 - 5 } & $t=0.003504$ & Order & $t=0.003505$ & Order \\
\hline $1024 \times 1024$ & $8.5671 \times 10^{-4}$ & - & $1.1352 \times 10^{-3}$ & - \\
$1280 \times 1280$ & $3.6084 \times 10^{-4}$ & 3.87 & $4.5801 \times 10^{-4}$ & 4.07 \\
$1536 \times 1536$ & $1.6929 \times 10^{-4}$ & 4.15 & $2.3050 \times 10^{-4}$ & 3.77 \\
$1792 \times 1792$ & $8.9837 \times 10^{-5}$ & 4.11 & $3.3212 \times 10^{-4}$ & $-^{\dagger}$ \\
\hline Sup-norm & $1.2209 \times 10^{11}$ & - & $1.2401 \times 10^{12}$ & - \\
\hline${ }^{\dagger}$ Round-off error begins to dominate.
\end{tabular}

physically important but is difficult to check in practice, because it requires selection and tracking of representative material curves, which is not always easy. On the other hand, in axisymmetric flows the total circulation along the circular contours

$$
C=\left\{(x, y, z) \in \mathbb{R}^{3}: x^{2}+y^{2}=r^{2}<1, z \text { a constant }\right\}
$$

is easily found to be $\Gamma=2 \pi r^{2} u_{1}$. Thus as an alternative to conservation of circulation, we choose to monitor the extreme circulations

$$
\Gamma_{1}=2 \pi \min _{(r, z) \in D(1, L)} r^{2} u_{1}(r, z), \quad \Gamma_{2}=2 \pi \max _{(r, z) \in D(1, L)} r^{2} u_{1}(r, z),
$$

which must be conserved over time according to Kelvin's circulation theorem.

We study the errors of the above-mentioned global quantities as follows. For conserved quantities such as kinetic energy and extreme circulations, the maximum (relative) change

$$
\|\delta Q\|_{\infty, t}=\max _{s \in[0, t]}|\delta Q(s)|
$$

Copyright $@$ by SIAM. Unauthorized reproduction of this article is prohibited. 
Table 7 Maximum (relative) change of kinetic energy $E$, minimum circulation $\Gamma_{1}$, and maximum circulation $\Gamma_{2}$ over the time interval [0,0.003505]. The initial value of each quantity, measured on the finest $2048 \times 2048$ mesh, is indicated in the last row of the table, "Init. value."

\begin{tabular}{cccc}
\hline \multirow{2}{*}{ Mesh size } & \multicolumn{3}{c}{$t=0.003505$} \\
\cline { 2 - 4 } & $\|\delta E\|_{\infty, t}$ & $\left\|\delta \Gamma_{1}\right\|_{\infty, t}$ & $\left\|\delta \Gamma_{2}\right\|_{\infty, t}$ \\
\hline $1024 \times 1024$ & $1.5259 \times 10^{-11}$ & $4.3525 \times 10^{-17}$ & $1.2485 \times 10^{-14}$ \\
$1280 \times 1280$ & $4.1730 \times 10^{-12}$ & $3.3033 \times 10^{-17}$ & $7.7803 \times 10^{-15}$ \\
$1536 \times 1536$ & $2.0787 \times 10^{-12}$ & $3.1308 \times 10^{-17}$ & $9.9516 \times 10^{-15}$ \\
$1792 \times 1792$ & $6.4739 \times 10^{-13}$ & $2.7693 \times 10^{-17}$ & $2.1351 \times 10^{-14}$ \\
$2048 \times 2048$ & $6.6594 \times 10^{-13}$ & $2.5308 \times 10^{-17}$ & $3.4921 \times 10^{-14}$ \\
\hline Init. value & 55.9309 & 0.0000 & $6.2832 \times 10^{2}$ \\
\hline
\end{tabular}

Table 8 Relative error of enstrophy $\mathcal{E}$, enstrophy production rate $\mathcal{E}_{p}$, and maximum vorticity $\|\omega\|_{\infty}$ at $t=0.003505$. The absolute size of each quantity, measured on the finest $2048 \times 2048$ mesh, is indicated in the last row of the table, "Ref. value."

\begin{tabular}{ccccccc}
\hline \multirow{2}{*}{ Mesh size } & $\mathcal{E}$ & Order & $\mathcal{E}_{p}$ & Order & $\|\omega\|_{\infty}$ & Order \\
\cline { 2 - 7 } & & - & $4.6565 \times 10^{-5}$ & - & $7.7593 \times 10^{-4}$ & - \\
\hline $1024 \times 1024$ & $4.6075 \times 10^{-6}$ & & - & \\
$1280 \times 1280$ & $1.4946 \times 10^{-6}$ & 5.05 & $1.4488 \times 10^{-5}$ & 5.23 & $3.0099 \times 10^{-4}$ & 4.24 \\
$1536 \times 1536$ & $5.6161 \times 10^{-7}$ & 5.37 & $5.3275 \times 10^{-6}$ & 5.49 & $1.2927 \times 10^{-4}$ & 4.64 \\
$1792 \times 1792$ & $2.3385 \times 10^{-7}$ & 5.68 & $2.0314 \times 10^{-6}$ & 6.25 & $6.1010 \times 10^{-5}$ & 4.87 \\
\hline Ref. value & $7.0254 \times 10^{5}$ & - & $1.4270 \times 10^{10}$ & - & $1.2401 \times 10^{12}$ & - \\
\hline
\end{tabular}

over the time interval $[0, t]$ is computed, where

$$
\delta Q(t)= \begin{cases}Q(0)^{-1}[Q(t)-Q(0)] & \text { if } Q(0) \neq 0 \\ Q(t)-Q(0) & \text { if } Q(0)=0\end{cases}
$$

For other nonconservative quantities, the relative error

$$
\frac{1}{\hat{Q}(t)}|Q(t)-\hat{Q}(t)|
$$

is computed where $Q$ denotes global quantities computed on a $256 k \times 256 k$ mesh and $\hat{Q}$ represents reference values obtained on the finer $[256(k+1)] \times[256(k+1)]$ mesh. The resulting errors and numerical orders at $t=0.003505$ are summarized in Tables $7-8$.

As a side remark, we note that the error of the maximum vorticity $\|\omega\|_{\infty}$ is always a lower bound of the error of the vorticity vector $\omega$. This is a direct consequence of the triangle inequality

$$
\left|\|\omega\|_{\infty}-\|\hat{\omega}\|_{\infty}\right| \leq\|\omega-\hat{\omega}\|_{\infty}
$$

and is readily confirmed by the results shown in Tables 6 and 8 . In addition, note that global errors such as the error of the enstrophy $\mathcal{E}$ can significantly underestimate the pointwise error of the vorticity vector $\omega$. This confirms the inadequacy of the "conventional" error indicators in the context of singularity detection. 


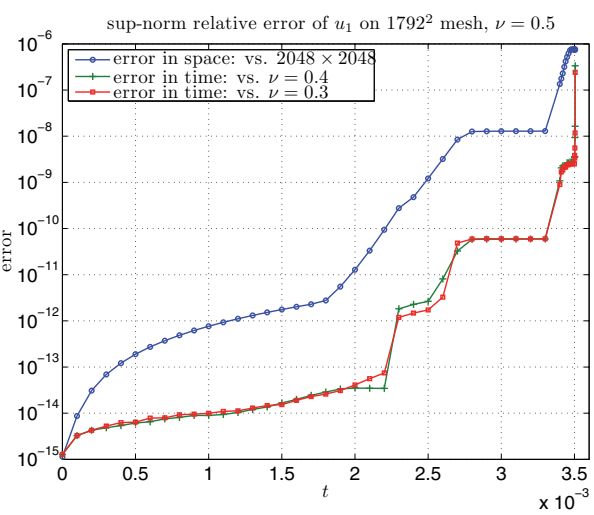

(a) sup-norm relative error of $u_{1}$

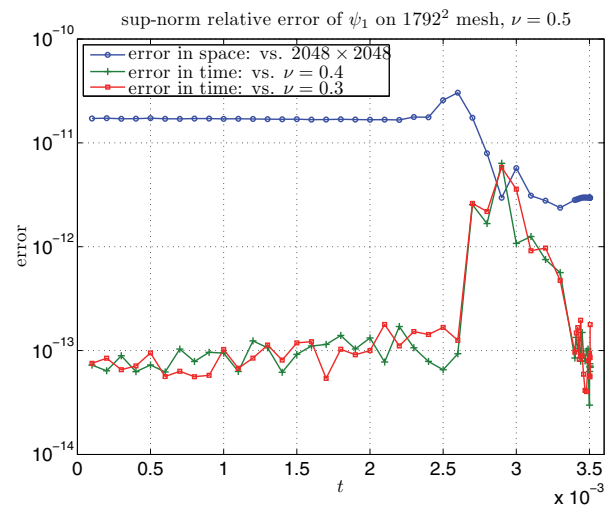

(c) sup-norm relative error of $\psi_{1}$

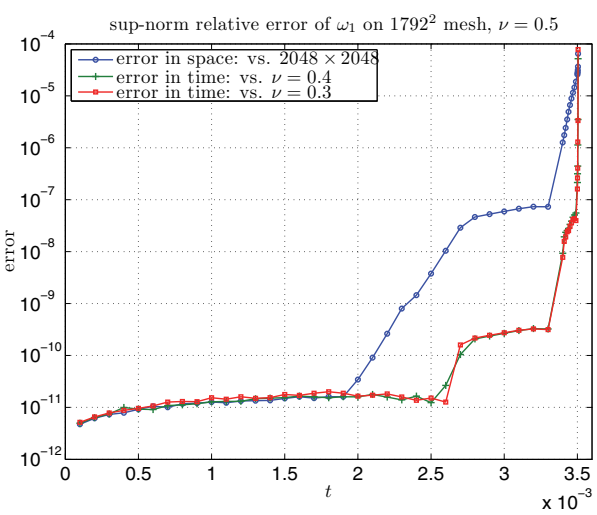

(b) sup-norm relative error of $\omega_{1}$

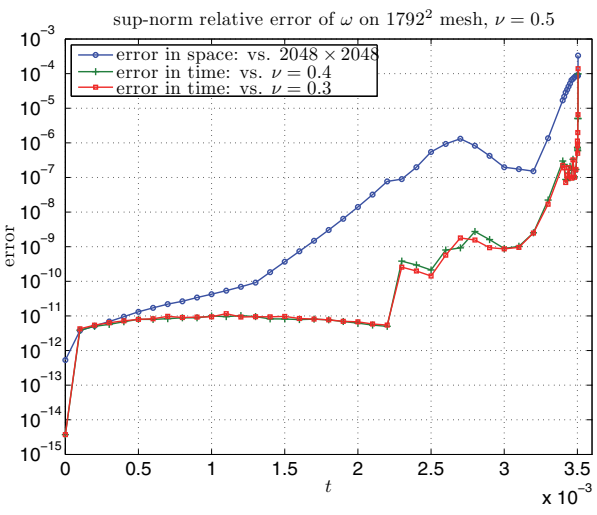

(d) sup-norm relative error of $\omega$

Fig. 5 Resolution study in time: sup-norm relative error of (a)-(c) the transformed primitive variables $\left(u_{1}, \omega_{1}, \psi_{1}\right)$ and $(\mathrm{d})$ the vorticity vector $\omega$, computed on the $1792 \times 1792$ mesh. The last time instant shown in the figure is $t=0.003505$.

4.3.5. Resolution Study in Time. Finally, we perform a resolution study in time by repeating the $1792 \times 1792$ mesh computation using smaller time steps $\delta_{t}$. This is achieved by reducing the CFL number from $\nu=0.5$ to $0.4,0.3$ and the relative growth threshold from $\epsilon_{t}=5 \%$ to $4 \%, 3 \%$ (section 3.3). For each reduced time step computation, the resulting solution $\left(\hat{u}_{1}, \hat{\omega}_{1}, \hat{\psi}_{1}, \hat{\omega}\right)$ is taken as the reference solution and is compared with the original solution $\left(u_{1}, \omega_{1}, \psi_{1}, \omega\right)$ computed using $\left(\nu, \epsilon_{t}\right)=(0.5,5 \%)$. The corresponding sup-norm errors are summarized in Figure 5 and Table 9. Note that the error between the computations $\left(\nu, \epsilon_{t}\right)=\{(0.3,3 \%),(0.5,5 \%)\}$ is roughly the same as that between the computations $\left(\nu, \epsilon_{t}\right)=\{(0.4,4 \%),(0.5,5 \%)\}$, which is smaller than the error between the $1792 \times 1792$ and the $2048 \times 2048$ mesh computations. This indicates that the solutions computed on the $1792 \times 1792$ and all the coarser meshes with $\left(\nu, \epsilon_{t}\right)=(0.5,5 \%)$ are well resolved in time up to $t=0.003505$.

4.4. Asymptotic Scaling Analysis I: Maximum Vorticity. With the pointwise error bounds derived in the previous section, we are ready to examine the numerical data in greater detail and apply the mathematical criteria reviewed in section 1 to assess the likelihood of a finite-time singularity. 
Table 9 Sup-norm relative error of the transformed primitive variables $\left(u_{1}, \omega_{1}, \psi_{1}\right)$ and the vorticity vector $\omega$, computed on the $1792 \times 1792$ mesh with $\left(\nu, \epsilon_{t}\right)=(0.5,5 \%)$ and compared with different reference solutions. The absolute size of each variable, measured on the finest $2048 \times 2048$ mesh, is indicated in the last row of the table, "Sup-norm."

\begin{tabular}{ccccc}
\hline \multirow{2}{*}{ Ref. solution } & \multicolumn{4}{c}{ Sup-norm relative error at $t=0.003505$} \\
\cline { 2 - 5 } & $u_{1}$ & $\omega_{1}$ & $\psi_{1}$ & $\omega$ \\
\hline $2048 \times 2048$ & $7.5561 \times 10^{-7}$ & $6.4388 \times 10^{-5}$ & $2.9518 \times 10^{-12}$ & $3.3212 \times 10^{-4}$ \\
$\left(\nu, \epsilon_{t}\right)=(0.4,4 \%)$ & $3.3350 \times 10^{-7}$ & $5.1609 \times 10^{-5}$ & $6.8713 \times 10^{-14}$ & $9.7514 \times 10^{-5}$ \\
$\left(\nu, \epsilon_{t}\right)=(0.3,3 \%)$ & $2.4197 \times 10^{-7}$ & $7.7720 \times 10^{-5}$ & $1.7776 \times 10^{-13}$ & $1.3800 \times 10^{-4}$ \\
\hline Sup-norm & $1.0000 \times 10^{2}$ & $1.0877 \times 10^{6}$ & $2.1610 \times 10^{-1}$ & $1.2401 \times 10^{12}$ \\
\hline
\end{tabular}

The basic tool that we shall employ is the well-known Beale-Kato-Majda (BKM) criterion [4]. According to this criterion, a smooth solution of the 3D Euler equations blows up at time $t_{s}$ if and only if

$$
\int_{0}^{t_{s}}\|\omega(\cdot, t)\|_{\infty} d t=\infty
$$

where $\|\omega(\cdot, t)\|_{\infty}$ is the maximum vorticity of the flow at time $t$. The BKM criterion was originally proved in [4] for flows in free space $\mathbb{R}^{3}$, and it was later generalized by $[32,64]$ to flows in smooth bounded domains subject to no-flow, free-slip boundary conditions. In view of this criterion, a "standard" approach to singularity detection in Euler computations is to assume the existence of an appropriate asymptotic scaling for $\|\omega\|_{\infty}$, typically in the form of an inverse power law

$$
\|\omega(\cdot, t)\|_{\infty} \sim c\left(t_{s}-t\right)^{-\gamma}, \quad c, \gamma>0
$$

then an estimate of the (unknown) singularity time $t_{s}$ and the scaling parameters $(c, \gamma)$ is obtained from a line fitting procedure. Normally, the line fitting is computed on some interval $\left[\tau_{1}, \tau_{2}\right]$ prior to the predicted singularity time $t_{s}$, and the results are extrapolated forward in time to yield the desired estimates.

Although seemingly straightforward, the above procedure must be applied with great caution. Indeed, there are examples where inadvertent line fitting has led to false predictions of finite-time singularities. As we shall demonstrate below, the key to the successful application of the line fitting procedure lies in the choice of the fitting interval $\left[\tau_{1}, \tau_{2}\right]$. One must realize, upon the invocation of (4.2), that the applicability of this form fit is not known a priori and must be determined from the line fitting itself. In order for the line fitting to work, the interval $\left[\tau_{1}, \tau_{2}\right]$ must be placed within the asymptotic regime of (4.2) if scalings of that form indeed exist. If such an asymptotic regime cannot be identified, then the validity of (4.2) is questionable and any conclusions drawn from the line fitting are likely to be false.

In most existing studies, the choice of the fitting interval $\left[\tau_{1}, \tau_{2}\right]$ is based on discretionary manual selections, which tend to generate results that lack clear interpretations and that are difficult to reproduce. To overcome these difficulties, we propose to choose $\tau_{1}, \tau_{2}$ using an automated procedure, which in ideal situations should place $\tau_{2}$ at $t_{s}$ and $\tau_{1}$ at a point "close enough" to $t_{s}$, in such a way that $\left[\tau_{1}, \tau_{2}\right]$ is enclosed in the asymptotic regime of (4.2). In reality, such a choice can never be made, because a singularity time $t_{s}$, if it exists, can never be attained by a numerical simulation. Thus as a compromise, we propose to place $\tau_{1}, \tau_{2}$ close enough to the stopping time $t_{e}$, in 
such a way that the computed solutions are still "well resolved" on $\left[\tau_{1}, \tau_{2}\right]$ and that an asymptotic scaling of the form (4.2) exists and prevails in $\left[\tau_{1}, \tau_{2}\right]$. To this end, we shall choose $\tau_{2}$ to be the first time instant at which the sup-norm relative error of the vorticity vector $\omega$ exceeds a certain threshold $\epsilon_{2}$, and choose $\tau_{1}$ so that $\left[\tau_{1}, \tau_{2}\right]$ is the interval on which the line fitting yields the "best" results (in a sense to be made precise below). Note that the accuracy of the computed solutions is measured in terms of the error of $\omega$, not that of $\left(u_{1}, \omega_{1}, \psi_{1}\right)$, because $\omega$ is the quantity that controls the blowup.

We consider a line fitting "successful" if both $\tau_{2}$ and the line-fitting predicted singularity time $\hat{t}_{s}$ converge to the same finite value as the mesh is refined. The convergence should be monotone, i.e., $\tau_{2} \uparrow t_{s}, \hat{t}_{s} \downarrow t_{s}$, where $t_{s}$ is the common limit, the true singularity time. In addition, $\tau_{1}$ should converge to a finite value that is strictly less than $t_{s}$ as the mesh is refined. The reason that the convergence of $\tau_{2}, \hat{t}_{s}$ to the singularity time $t_{s}$ should be monotone is twofold: first, the finer the mesh, the longer it takes the error to grow to a given tolerance, and hence the larger the $\tau_{2}$ is; second, as $\tau_{2}$ gets increasingly closer to $t_{s}$, the strong, singular growth of the blowing-up solution is better captured on $\left[\tau_{1}, \tau_{2}\right]$, which then translates into an earlier estimate $\hat{t}_{s}$ of the blowup time.

If the interval $\left[\tau_{1}, \tau_{2}\right]$ can be chosen to satisfy all the above criteria, and the scaling parameters $(c, \gamma)$ estimated on this interval converge to some finite values $c_{s}>0, \gamma_{s} \geq 1$ as the mesh is refined, then the existence of a finite-time singularity is confirmed.

We apply the above ideas to our numerical data and estimate the parameters in (4.2) from the linear regression problems

$$
\begin{aligned}
& y(t):=\left\{\frac{d}{d t} \log \|\omega(\cdot, t)\|_{\infty}\right\}^{-1} \sim-\frac{1}{\gamma}\left(t-t_{s}\right)=: a t+b, \\
& \tilde{y}(\tilde{t}):=\log \|\omega(\cdot, t)\|_{\infty} \sim-\gamma \log \left(\hat{t}_{s}-t\right)+\log c=: \tilde{a} \tilde{t}+\tilde{b},
\end{aligned}
$$

where $\hat{t}_{s}$ is an estimate of the singularity time $t_{s}$ computed from (4.3), and

$$
a=-1 / \gamma, b=t_{s} / \gamma, \quad \text { and } \quad \tilde{a}=-\gamma, \tilde{b}=\log c, \tilde{t}=\log \left(\hat{t}_{s}-t\right) .
$$

The model parameters $(a, b, \tilde{a}, \tilde{b})$ of these regression problems are estimated from a standard least-squares procedure, and the fitness of the models is assessed using the fraction of variance unexplained (FVU), where a value of FVU close to 0 indicates good fitness. The fitting interval $\left[\tau_{1}, \tau_{2}\right]$ is chosen so that $\tau_{2}$ is the first time instant at which the sup-norm relative error of $\omega$ exceeds a certain threshold $\epsilon_{2}$, and $\tau_{1}$ is the time instant at which the FVU of the line fitting computed on $\left[t, \tau_{2}\right]$, when viewed as a function of $t$, attains a local minimum. The detailed description of the algorithm, as well as the precise definition of the quality measure FVU, can be found in [58, sections 4.4.1-4.4.2].

To see whether the above procedure applies to the maximum vorticity $\|\omega\|_{\infty}$, we first plot in Figure 6 the inverse $\log t$-derivative of the maximum vorticity (cf. (4.3))

$$
y(t)=\left\{\frac{d}{d t} \log \|\omega(\cdot, t)\|_{\infty}\right\}^{-1}
$$

computed on the $2048 \times 2048$ mesh. Intuitively, the inverse log $t$-derivative approaches a straight line after $t \approx 0.0032$, which suggests that the maximum vorticity indeed admits an inverse power law of the form (4.2). Motivated by this observation, we 


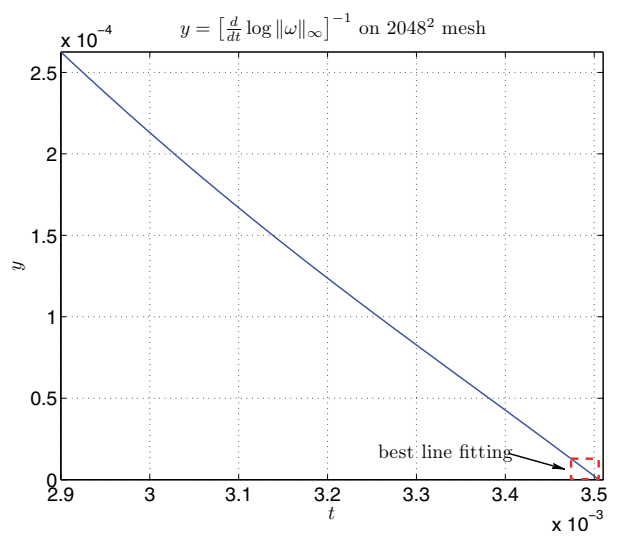

Fig. 6 Inverse log t-derivative of the maximum vorticity computed on the $2048 \times 2048$ mesh. The dashed line box represents the best fitting interval $\left[\tau_{1}, \tau_{2}\right]$.

Table 10 The best line fittings (4.3) and (4.4) computed on the interval $\left[\tau_{1}, \tau_{2}\right]$ with $n$ data points.

\begin{tabular}{cccccccc}
\hline Mesh size & $n$ & $\tau_{1}$ & $\tau_{2}$ & $\hat{t}_{s}$ & $\hat{\gamma}_{1}$ & $\hat{\gamma}_{2}$ & $\hat{c}$ \\
\hline $1024 \times 1024$ & 58 & 0.003306 & 0.003410 & 0.0035070 & 2.5041 & 2.5062 & $4.8293 \times 10^{-4}$ \\
$1280 \times 1280$ & 47 & 0.003407 & 0.003453 & 0.0035063 & 2.4866 & 2.4894 & $5.5362 \times 10^{-4}$ \\
$1536 \times 1536$ & 20 & 0.003486 & 0.003505 & 0.0035056 & 2.4544 & 2.4559 & $7.4912 \times 10^{-4}$ \\
$1792 \times 1792$ & 27 & 0.003479 & 0.003505 & 0.0035056 & 2.4557 & 2.4566 & $7.4333 \times 10^{-4}$ \\
$2048 \times 2048$ & 32 & 0.003474 & 0.003505 & 0.0035056 & 2.4568 & 2.4579 & $7.3273 \times 10^{-4}$ \\
\hline
\end{tabular}

apply the line fitting to the data $y$ and report the resulting estimates in Table 10 . It can be observed from this table that all estimated parameters converge to a finite limit as the mesh is refined, where in particular both $\tau_{2}$ and $\hat{t}_{s}$ tend to a common limit in a monotonic fashion. ${ }^{8}$ In addition, it can be seen that the limit of $\tau_{1}$ is strictly less than the common limit of $\tau_{2}$ and $\hat{t}_{s}$, indicating the existence of an asymptotic regime. Moreover, both estimates $\hat{\gamma}_{1}, \hat{\gamma}_{2}$ of $\gamma$ (computed from (4.3) and (4.4), respectively) approach a common limit with a value close to $\frac{5}{2} \geq 1$, and the limit of $\hat{c}$ is strictly positive. Based on these observations and the BKM criterion, we conclude that the solution of problem (2.1)-(2.2) develops a singularity at $t_{s} \approx 0.0035056$.

It is interesting to compare at this point the two estimates $\hat{\gamma}_{1}, \hat{\gamma}_{2}$ of the scaling exponent $\gamma$ computed from the regression problems (4.3) and (4.4). As can be observed from Table 10, the estimate $\hat{\gamma}_{2}$ computed from (4.4) is always slightly larger than that $\hat{\gamma}_{1}$ computed from (4.3). This is expected, because the singularity time $\hat{t}_{s}$ estimated from (4.3) decreases monotonically as the mesh is refined, indicating that $\hat{t}_{s}$ is always an overestimate of the true singularity time $t_{s}$. Consequently, the inverse power law $\left(\hat{t}_{s}-t\right)^{-\gamma}$ necessarily underestimates the maximum vorticity $\|\omega\|_{\infty} \sim\left(t_{s}-t\right)^{-\gamma}$ when $t$ is sufficiently close to $t_{s}$, and the scaling exponent $\hat{\gamma}_{2}$ estimated from (4.4) has to be artificially magnified to compensate for this discrepancy. This explains the larger value of $\hat{\gamma}_{2}$ compared with $\hat{\gamma}_{1}$.

\footnotetext{
${ }^{8}$ The small discrepancy between the limits of $\tau_{2}$ and $\hat{t}_{s}$ is due to the fact that the sup-norm errors of $\omega$ are computed only at a discrete set of time instants. This restricts the definition of $\tau_{2}$ to a discrete set of values.
} 
Table II Comparison of our results with other representative numerical studies. $\tau_{2}$ : the last time at which the solution is deemed "well resolved."

\begin{tabular}{ccccc}
\hline Studies & $\tau_{2}$ & $t_{s}$ & Effec. res. & Vort. amp. \\
\hline$[50]$ & 17 & 18.7 & $\leq 512^{3}$ & 23 \\
{$[7]$} & $1.6^{\dagger}$ & 2.06 & $1024^{3}$ & 180 \\
{$[37]$} & 1.32 & 1.355 & $2048^{3}$ & 21 \\
{$[61]$} & 2.72 & 2.75 & $1024^{3}$ & 55 \\
Ours & 0.003505 & 0.0035056 & $\left(3 \times 10^{12}\right)^{2}$ & $3 \times 10^{8}$ \\
\hline
\end{tabular}

${ }^{\dagger}$ According to [45].

The computation of $\hat{\gamma}_{1}$ from (4.3), on the other hand, does not suffer from this problem and is expected to yield a more accurate estimate of $\gamma$. Thus in what follows, we shall always choose $\hat{\gamma}_{1}$ as the estimated value of $\gamma$.

The quality of the line fittings computed in Table 10 can be assessed using several metrics, including the FVU computed on the fitting interval $\left[\tau_{1}, \tau_{2}\right]$, the "extrapolated FVU" computed on the extrapolation interval $\left[\tau_{2}, t_{e}\right]$, and the errors of the line fittings computed on the extrapolation interval $\left[\tau_{2}, t_{e}\right]$. The results, as reported in [58, section 4.4.3], demonstrate that both linear models (4.3) and (4.4) fit the data very well, and that the line fittings provide an excellent approximation to the data even in the extrapolation interval. Based on these observations, we conclude that the estimates obtained in Table 10 are trustworthy.

We conclude this section with a brief comparison of our results with other representative numerical studies (Table 11). As is clear from the table, our computation offers a much higher effective mesh resolution, and it advances the solution to a point that is significantly closer to the time predicted for singularity formation. It also produces a much stronger vorticity amplification, thus yielding much more convincing evidence for the existence of a finite-time singularity compared with other numerical studies.

4.5. Asymptotic Scaling Analysis II: Vorticity Moments. Given the existence of a finite-time singularity as indicated by the blowing-up maximum vorticity $\|\omega\|_{\infty}$, we turn to the interesting question of whether the vorticity moment integrals

$$
\Omega_{2 m}=\left(\int_{D(1, L)}|\omega|^{2 m} d x\right)^{1 / 2 m}, \quad m=1,2, \ldots,
$$

blow up at the same time as $\|\omega\|_{\infty}$ does, and if so, what type of asymptotic scalings they satisfy. According to Hölder's inequality, higher vorticity moments "control" the growth of lower vorticity moments, in the sense that

$$
\Omega_{2 m} \leq \Omega_{2 n}|D(1, L)|^{(n-m) /(2 m n)}, \quad 1 \leq m<n .
$$

Thus the blowup of any vorticity moment $\Omega_{2 m}$ implies the blowup of all higher moments $\Omega_{2 n}(n \geq m)$. In particular, since $\|\omega\|_{\infty}=\Omega_{\infty}$, the blowup of any finite-order vorticity moment implies the blowup of the maximum vorticity, hence providing additional supporting evidence for the existence of a finite-time singularity.

We have carried out a detailed analysis of the vorticity moments and discovered that all moments of order higher than 2 blow up at a finite time. ${ }^{9}$ For the purpose of

\footnotetext{
${ }^{9}$ The enstrophy integral, $\Omega_{2}^{2}$, is observed to grow rapidly (faster than double-exponential), but careful analysis indicates that it is likely to remain bounded as the singularity time $t_{s}$ is approached.
} 
Table 12 The line fitting (4.5) of the 2 th vorticity moment $\Omega_{2 m}, m=2,3,4$, computed on the interval $\left[\tau_{1}, \tau_{2}\right]$. For comparison, the singularity time $\hat{t}_{s}$ estimated from (4.3) is also included.

\begin{tabular}{cccccccc}
\hline \multirow{2}{*}{ Mesh size } & \multirow{2}{*}{$\hat{t}_{s}$ from $(4.3)$} & \multicolumn{3}{c}{$\hat{t}_{2 m, s}$} & \multicolumn{3}{c}{$\hat{\gamma}_{2 m, 1}$} \\
\cline { 3 - 8 } & & $m=2$ & $m=3$ & $m=4$ & $m=2$ & $m=3$ & $m=4$ \\
\hline $1024 \times 1024$ & 0.0035070 & 0.0035231 & 0.0035124 & 0.0035097 & 1.2542 & 1.6129 & 1.8176 \\
$1280 \times 1280$ & 0.0035063 & 0.0035115 & 0.0035074 & 0.0035067 & 1.1306 & 1.5383 & 1.7658 \\
$1536 \times 1536$ & 0.0035056 & 0.0035056 & 0.0035056 & 0.0035056 & 1.0019 & 1.4857 & 1.7289 \\
$1792 \times 1792$ & 0.0035056 & 0.0035057 & 0.0035056 & 0.0035056 & 1.0039 & 1.4855 & 1.7285 \\
$2048 \times 2048$ & 0.0035056 & 0.0035057 & 0.0035056 & 0.0035056 & 1.0062 & 1.4857 & 1.7285 \\
\hline
\end{tabular}

illustration, we report in Table 12 the singularity time $\hat{t}_{2 m, s}$ and the scaling exponent $\hat{\gamma}_{2 m, 1}$ estimated from the line fitting

$$
y(t):=\left\{\frac{d}{d t} \log \Omega_{2 m}(t)\right\}^{-1} \sim-\frac{1}{\gamma_{2 m}}\left(t-t_{s}\right)=: a t+b
$$

for $m=2,3,4$, where $\Omega_{2 m}$ is assumed to satisfy the scaling law

$$
\Omega_{2 m}(t) \sim c_{2 m}\left(t_{s}-t\right)^{-\gamma_{2 m}}, \quad c_{2 m}, \gamma_{2 m}>0 .
$$

It can be observed from this table that all $\Omega_{2 m}$ with $m>1$ satisfy an inverse power law, with an exponent $\hat{\gamma}_{2 m, 1}$ monotonically approaching $\hat{\gamma} \approx \frac{5}{2}$, and that they all blow up at a finite time $\hat{t}_{2 m, s}$ approximately equal to the singularity time $\hat{t}_{s}$ estimated from (4.3). This confirms the blowup of $\|\omega\|_{\infty}$ at the predicted singularity time $t_{s}$, and hence the existence of a finite-time singularity.

4.6. Vorticity Direction and Spectral Dynamics. The BKM criterion characterizes the finite-time blowup of the 3D Euler equations in terms of the maximum vorticity magnitude $|\omega|$, but makes no assumption on the vorticity direction $\xi=\omega /|\omega|$. It turns out that, when less regularity is demanded for the vorticity magnitude, say boundedness in $L^{p}(p<\infty)$ instead of boundedness in $L^{\infty}$, the regularity of the vorticity direction can also play a role in controlling the blowup of the Euler solutions [23]. To see more precisely how the direction vector $\xi$ enters the analysis, recall the vorticity amplification equation

$$
\begin{gathered}
|\omega|_{t}+u \cdot \nabla|\omega|=\alpha|\omega|, \\
\alpha=\xi \cdot \nabla u \cdot \xi=\xi \cdot S \xi, \quad S=\frac{1}{2}\left(\nabla u+\nabla u^{T}\right),
\end{gathered}
$$

which can be derived from (1.2) by applying the scalar product $\omega$. on both sides of the equation. The vorticity amplification factor $\alpha$ can be shown to admit the representation [23]:

$$
\alpha(x)=\frac{3}{4 \pi} \mathrm{P} \cdot \mathrm{V} \cdot \int_{\mathbb{R}^{3}} D(\hat{y}, \xi(x+y), \xi(x))|\omega(x+y)| \frac{d y}{|y|^{3}},
$$

where $\hat{y}=y /|y|$ is the unit vector pointing in the direction of $y$ and

$$
D\left(e_{1}, e_{2}, e_{3}\right)=\left(e_{1} \cdot e_{3}\right) \operatorname{det}\left(e_{1}, e_{2}, e_{3}\right) .
$$

Note that the quantity $D\left(e_{1}, e_{2}, e_{3}\right)$ is small when $e_{2}$ and $e_{3}$ are nearly aligned or antialigned, so a smoothly varying vorticity direction field $\xi$ near a spatial point $x$ 


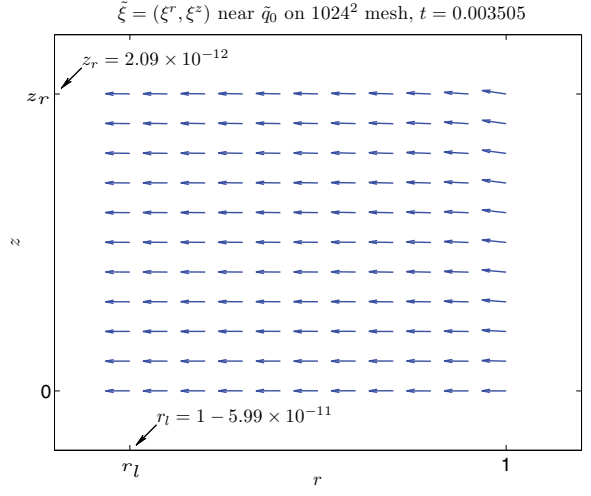

(a) 2D vorticity direction $\tilde{\xi}=\left(\xi^{r}, \xi^{z}\right)^{T}$

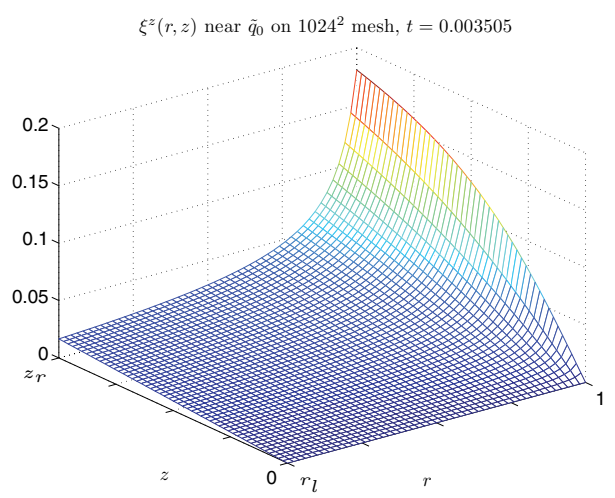

(b) $z$-component $\xi^{z}$ of vorticity direction

Fig. 7 The geometry of the vorticity direction: (a) the $2 D$ vorticity direction $\tilde{\xi}=\left(\xi^{r}, \xi^{z}\right)^{T}$ and (b) the $z$-direction component $\xi^{z}$ computed on the $1024 \times 1024$ mesh at $t=0.003505$. All plots in this figure are defined on the region $\left[r_{l}, 1\right] \times\left[0, z_{r}\right]$, where $r_{l}=1-5.99 \times 10^{-11}$ and $z_{r}=2.09 \times 10^{-12}$.

can induce strong cancellation in the vorticity amplification $\alpha(x)$, thus preventing the vorticity $|\omega(x)|$ at $x$ from growing unboundedly. The most well-known nonblowup criteria in this direction are those of Constantin, Fefferman, and Majda [25] and Deng, $\mathrm{Hou}$, and $\mathrm{Yu}[26]$. Under the assumption that the vorticity direction $\xi$ is "not too twisted" near the location of the maximum vorticity, they show that a suitable upper bound can be obtained for $\alpha$ and hence for $\|\omega\|_{\infty}$, establishing the regularity of the solutions to the 3D Euler equations.

The Constantin-Fefferman-Majda (CFM) and Deng-Hou-Yu (DHY) nonblowup criteria are useful for excluding false blowup candidates, but they cannot be used directly to verify a finite-time singularity. The reason is that these criteria provide only upper bounds for the amplification factor $\alpha$, while a blowup estimate requires a lower bound. Nevertheless, a careful examination of our numerical data against these criteria provides additional evidence for the existence of a finite-time singularity. It also offers additional insights into the nature of the blowup.

By carefully checking our numerical data against the CFM and DHY criteria, we confirm, as shown in [58, sections 4.6.1-4.6.2], that the conditions stated in these theorems are not satisfied; as a result, none of the criteria excludes the possibility of a finite-time singularity from our data. To better appreciate the local geometric structure of the vorticity direction $\xi$ near the location of the maximum vorticity, we also include in Figure 7 a plot of the 2D vorticity direction $\tilde{\xi}=\left(\xi^{r}, \xi^{z}\right)^{T}$ and a plot of the $z$-direction component $\xi^{z}$, both defined at $t=0.003505$ on the set

$$
\tilde{D}_{\infty}=\left[1-5.99 \times 10^{-11}, 1\right] \times\left[0,2.09 \times 10^{-12}\right] .
$$

Note that the through-plane $(\theta)$ component of $\xi$ has a maximum absolute value of $2.1874 \times 10^{-6}$ in $\tilde{D}_{\infty}$ and hence is negligible there. It can also be observed from Figure 7 that the $z$-direction component $\xi^{z}$ experiences an $O(1)$ change in $\tilde{D}_{\infty}$ along the $z$-dimension, which has a length scale of order $O\left(10^{-12}\right)$. This indicates the formation of bundles of "densely packed" and "fast twisting" vortex lines near the location of the maximum vorticity, hence explaining the breakdown of the smooth vorticity direction $\xi$ and that of the nonblowup criteria in that region. 
The analysis carried out above suggests that the growth of the vorticity amplification factor $\alpha$ depends on the local geometric structures of the vorticity vector. Alternatively, the dynamics of the vorticity amplification can also be investigated algebraically, using the defining relation (cf. (4.6b))

$$
\alpha=\xi \cdot \nabla u \cdot \xi=\xi \cdot S \xi, \quad S=\frac{1}{2}\left(\nabla u+\nabla u^{T}\right)
$$

where the eigenstructure of the symmetric strain tensor $S$ plays a central role. To see more clearly the connection between $\alpha$ and $S$, consider the velocity vector in cylindrical coordinates:

$$
u=u^{r} e_{r}+u^{\theta} e_{\theta}+u^{z} e_{z}
$$

where the three Cartesian components of $u$ can be expressed in terms of the transformed variables $\left(u_{1}, \psi_{1}\right)$ :

$$
\begin{aligned}
& v_{1}=-r \psi_{1, z} \cos \theta-r u_{1} \sin \theta, \\
& v_{2}=-r \psi_{1, z} \sin \theta+r u_{1} \cos \theta, \\
& v_{3}=2 \psi_{1}+r \psi_{1, r} .
\end{aligned}
$$

The entries of the deformation tensor $\nabla u$ can be readily computed:

$$
\begin{array}{lll}
\left.\partial_{x} v_{1}\right|_{\theta=0}=-\psi_{1, z}-r \psi_{1, r z}, & \left.\partial_{y} v_{1}\right|_{\theta=0}=-u_{1}, & \left.\partial_{z} v_{1}\right|_{\theta=0}=-r \psi_{1, z z} \\
\left.\partial_{x} v_{2}\right|_{\theta=0}=u_{1}+r u_{1, r}, & \left.\partial_{y} v_{2}\right|_{\theta=0}=-\psi_{1, z}, & \left.\partial_{z} v_{2}\right|_{\theta=0}=r u_{1, z} \\
\left.\partial_{x} v_{3}\right|_{\theta=0}=3 \psi_{1, r}+r \psi_{1, r r}, & \left.\partial_{y} v_{3}\right|_{\theta=0}=0, & \left.\partial_{z} v_{3}\right|_{\theta=0}=2 \psi_{1, z}+r \psi_{1, r z}
\end{array}
$$

where the computation need only be done on the meridian plane $\theta=0$ thanks to the axial symmetry. When further restricted to the point $\tilde{q}_{0}=(1,0)^{T}$, the location of the maximum vorticity, the above expressions reduce to

$$
\nabla \tilde{u}=\left(\begin{array}{ccc}
-\tilde{\psi}_{1, r z} & 0 & 0 \\
0 & 0 & \tilde{u}_{1, z} \\
0 & 0 & \tilde{\psi}_{1, r z}
\end{array}\right), \quad \tilde{S}=\left(\begin{array}{ccc}
-\tilde{\psi}_{1, r z} & 0 & 0 \\
0 & 0 & \frac{1}{2} \tilde{u}_{1, z} \\
0 & \frac{1}{2} \tilde{u}_{1, z} & \tilde{\psi}_{1, r z}
\end{array}\right)
$$

where for simplicity we have written $\nabla \tilde{u}=\nabla u\left(\tilde{q}_{0}\right), \tilde{S}=S\left(\tilde{q}_{0}\right)$, etc. Now the eigenvalues of $\tilde{S}$ can be easily found to be

$$
\tilde{\lambda}_{1,3}=\frac{1}{2}\left\{\tilde{\psi}_{1, r z} \pm\left(\tilde{\psi}_{1, r z}^{2}+\tilde{u}_{1, z}^{2}\right)^{1 / 2}\right\}, \quad \tilde{\lambda}_{2}=-\tilde{\psi}_{1, r z}
$$

with corresponding eigenvectors

$$
\tilde{w}_{1}=\left(\begin{array}{c}
0 \\
\frac{1}{2} \tilde{u}_{1, z} \\
\tilde{\lambda}_{1}
\end{array}\right), \quad \tilde{w}_{2}=\left(\begin{array}{c}
1 \\
0 \\
0
\end{array}\right), \quad \tilde{w}_{3}=\left(\begin{array}{c}
0 \\
\frac{1}{2} \tilde{u}_{1, z} \\
\tilde{\lambda}_{3}
\end{array}\right) .
$$

On the other hand, the vorticity vector $\omega$ at $\tilde{q}_{0}$ takes the form

$$
\tilde{\omega}=\left(\begin{array}{c}
-\tilde{u}_{1, z} \\
0 \\
0
\end{array}\right), \quad \text { with } \quad \tilde{\xi}=\frac{\tilde{\omega}}{|\tilde{\omega}|}=\left(\begin{array}{c}
-1 \\
0 \\
0
\end{array}\right) .
$$

Copyright $@$ by SIAM. Unauthorized reproduction of this article is prohibited. 
Thus the vorticity direction $\tilde{\xi}$ at the location of the maximum vorticity is perfectly aligned with $\tilde{w}_{2}$, the second eigenvector of $\tilde{S}$, and the corresponding amplification factor becomes

$$
\alpha_{\infty}:=\tilde{\alpha}=\tilde{\xi} \cdot \tilde{S} \tilde{\xi}=\tilde{\lambda}_{2}=-\tilde{\psi}_{1, r z} .
$$

It is worth noting that, when viewed in $\mathbb{R}^{3}$, the eigenvectors $\left\{\tilde{w}_{1}, \tilde{w}_{2}, \tilde{w}_{3}\right\}$ restricted to the "singularity ring"

$$
C=\left\{(x, y, z) \in \mathbb{R}^{3}: x^{2}+y^{2}=1, z=0\right\}
$$

form an orthogonal frame, with $\tilde{w}_{2}$ pointing in the radial direction and $\tilde{w}_{1}, \tilde{w}_{3}$ pointing in directions tangential to the lateral surface of the cylinder $r=1$.

Finally, by making use of the relations (cf. (4.6a))

$$
\alpha_{\infty}=-\tilde{\psi}_{1, r z}=\frac{d}{d t} \log \|\omega\|_{\infty}, \quad\|\omega\|_{\infty}=|\tilde{\omega}|=\left|\tilde{u}_{1, z}\right|,
$$

we may also express the first and third eigenvalues of $\tilde{S}$ in the form

$$
\tilde{\lambda}_{1,3}=\frac{1}{2}\left\{-\alpha_{\infty} \pm\left(\alpha_{\infty}^{2}+\|\omega\|_{\infty}^{2}\right)^{1 / 2}\right\} .
$$

Since $\alpha_{\infty}$ and $\|\omega\|_{\infty}$ both satisfy an inverse power law with an exponent roughly equal to -1 (for $\alpha_{\infty}$; cf. (4.3)) and $-\frac{5}{2}$ (for $\|\omega\|_{\infty}$ ), it follows that

$$
\tilde{\lambda}_{1,3} \sim \pm \frac{1}{2}\|\omega\|_{\infty}, \quad t \rightarrow t_{s}^{-} .
$$

This is confirmed by a line fitting similar to (4.4), which yields (cf. Table 10)

$$
\begin{array}{ll}
\tilde{\lambda}_{1} \sim c_{1}\left(\hat{t}_{s}-t\right)^{-2.4582}, & c_{1}=3.6514 \times 10^{-4}, \\
\tilde{\lambda}_{3} \sim c_{3}\left(\hat{t}_{s}-t\right)^{-2.4576}, & c_{3}=-3.6759 \times 10^{-4},
\end{array}
$$

where $\hat{t}_{s}$ is the singularity time estimated from (4.3).

4.7. Locally Self-Similar Structure. The 3D Euler equations (1.1) possess the well-known scaling property that if $u(x, t)$ is a solution of the equations, then

$$
u_{\lambda}(x, t):=\lambda^{\alpha} u\left(\lambda x, \lambda^{\alpha+1} t\right) \quad \forall \lambda>0, \forall \alpha \in \mathbb{R}
$$

is also a solution. A natural question is then whether the 3D Euler equations admit self-similar solutions of the form

$$
u(x, t)=\frac{1}{[T-t]^{\gamma}} U\left(\frac{x-x_{0}}{[T-t]^{\beta}}\right),
$$

where $U$ is a self-similar velocity profile and $\beta, \gamma$ are scaling exponents. By substituting (4.7a) into (1.1), it is easily found that

$$
\beta=\frac{1}{\alpha+1}, \quad \gamma=\frac{\alpha}{\alpha+1} \quad \forall \alpha \neq-1,
$$

which in particular implies that

$$
\nabla u(x, t)=\frac{1}{T-t} \nabla U\left(\frac{x-x_{0}}{[T-t]^{\beta}}\right) .
$$

Copyright $@$ by SIAM. Unauthorized reproduction of this article is prohibited. 
In $[13,14]$, the existence of global self-similar solutions of the form $(4.7 \mathrm{c})$ is excluded under one of the following conditions: either [14]

$$
\limsup _{t \rightarrow T^{-}}(T-t)\|\nabla u(\cdot, t)\|_{\infty}=\|\nabla U\|_{\infty}<1
$$

or $[13]$

$$
\Omega:=\nabla \times U \neq 0 \quad \text { and } \quad \Omega \in L^{p}\left(\mathbb{R}^{3}\right) \quad \forall p \in\left(0, p_{1}\right)
$$

for some $p_{1}>0$. Note that the first condition is not easy to interpret physically, while the second is too strong, effectively requiring that $\Omega$ decay exponentially fast at infinity or have compact support. These nonexistence results were generalized later in [14] to $\alpha$-asymptotically global self-similar solutions $\bar{U}$ of the form

$$
\lim _{t \rightarrow T^{-}}[T-t]^{1-(3 \beta / p)}\left\|\nabla u(\cdot, t)-\frac{1}{T-t} \nabla \bar{U}\left(\frac{\cdot-x_{0}}{[T-t]^{\beta}}\right)\right\|_{L^{p}\left(\mathbb{R}^{3}\right)}=0
$$

where $\beta=(\alpha+1)^{-1}$ and $p \in(0, \infty]$. Similar nonexistence results for local self-similar solutions were also obtained in [15].

In axisymmetric flows, self-similar solutions ${ }^{10}$ naturally take the form

$$
\begin{aligned}
& u_{1}(\tilde{x}, t) \sim[T-t]^{\gamma_{u}} U\left(\frac{\tilde{x}-\tilde{x}_{0}}{[T-t]^{\gamma_{l}}}\right), \\
& \omega_{1}(\tilde{x}, t) \sim[T-t]^{\gamma_{\omega}} \Omega\left(\frac{\tilde{x}-\tilde{x}_{0}}{[T-t]^{\gamma_{l}}}\right), \\
& \psi_{1}(\tilde{x}, t) \sim[T-t]^{\gamma_{\psi}} \Psi\left(\frac{\tilde{x}-\tilde{x}_{0}}{[T-t]^{\gamma_{l}}}\right), \quad \tilde{x} \rightarrow \tilde{x}_{0}, t \rightarrow T^{-},
\end{aligned}
$$

where $\tilde{x}=(r, z)^{T}$ is a point on the $r z$-plane and $(U, \Omega, \Psi)$ are self-similar profiles. Note that this ansatz does not correspond to a Leray-type self-similar solution (which contracts to a single point in $\mathbb{R}^{3}$; cf. (4.7a)) when viewed in $\mathbb{R}^{3}$; rather, it describes a tube-like anisotropic singularity due to the axial symmetry. In addition, the ansatz induces a scaling law (see section 4.7.3)

$$
\|\nabla u(\cdot, t)\|_{\infty}=O(T-t)^{\min \left\{\gamma_{u}-\gamma_{\ell},-1\right\}},
$$

which is very different from the "standard" law $\|\nabla u(\cdot, t)\|_{\infty}=O(T-t)^{-1}$ assumed by the nonexistence theorems $[13,14,15]$. In view of these observations, it is desirable to examine more closely the blowup solutions discovered in previous sections and look for clues for the existence of a (meridian-plane) self-similar solution. ${ }^{11}$

In what follows, we shall carry out a careful study of the numerical solution near the location of the maximum vorticity $\tilde{q}_{0}=(1,0)^{T}$ and demonstrate the existence of a locally self-similar blowup. By applying a line fitting similar to (4.4), we also deduce the scaling law $\|\nabla u(\cdot, t)\|_{\infty}=O(T-t)^{-2.4529}$ satisfied by the self-similar solution, confirming again the existence of a finite-time singularity.

\footnotetext{
${ }^{10}$ In what follows, whenever we say "self-similar solutions" for an axisymmetric flow, we always mean "self-similar solutions in the meridian plane."

${ }^{11}$ Note that in [17], two nonexistence results are proved which exclude the existence of nontrivial self-similar solutions of the form (4.8) to the 3D axisymmetric Euler equations under suitable decay conditions. It is to be observed that these results do not apply to the self-similar solutions described here, because the decay conditions are not satisfied.
} 


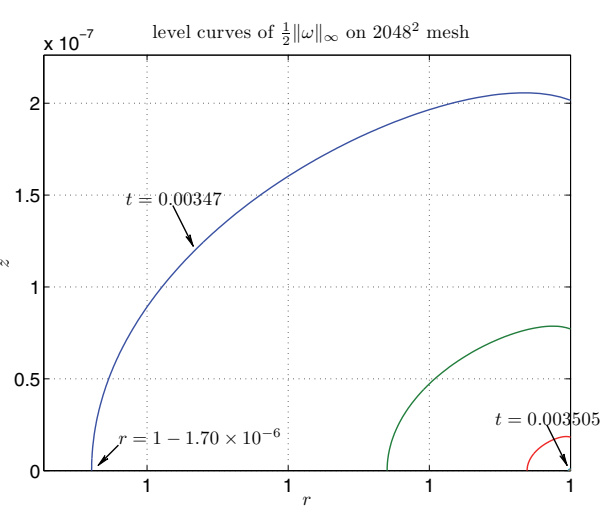

(a) linear-linear

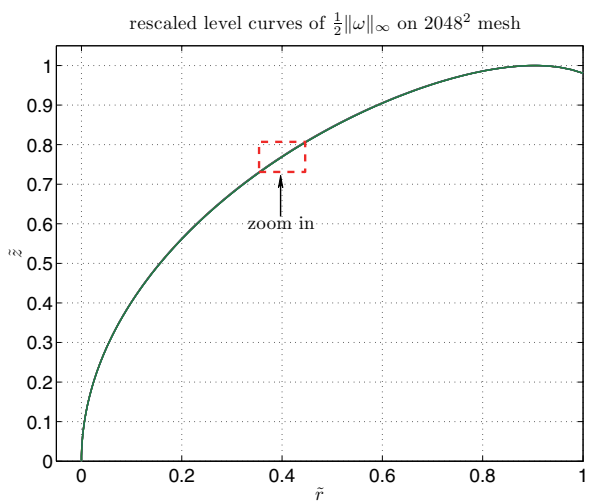

(c) rescaled

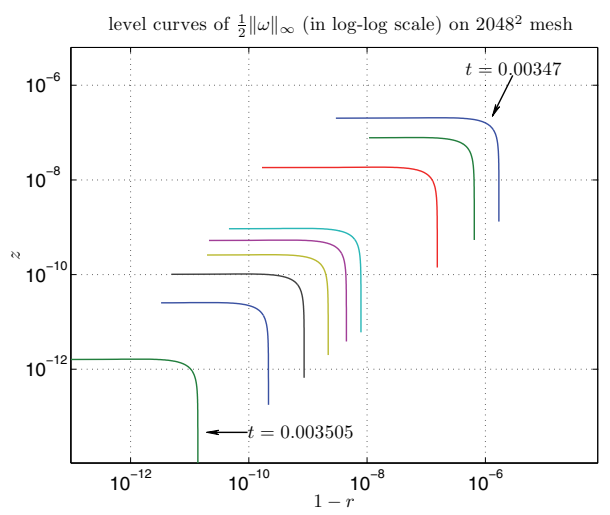

(b) $\log -\log$

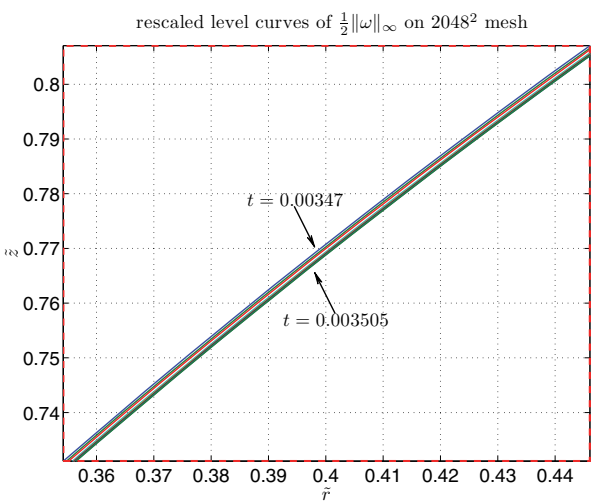

(d) rescaled (zoom-in)

Fig. 8 The level curves $C_{\infty}(t)$ at various time instants in (a) linear-linear and (b) log-log scale (against the variables $(1-r)$ and $z$ ). The rescaled level curves and their zoom-in view are shown in (c) and (d).

4.7.I. Existence of Self-Similar Neighborhood. The identification of a locally self-similar solution requires the identification of its three basic ingredients: first, the center of self-similarity, $\tilde{x}_{0}$, around which the self-similar structure is developed; second, a neighborhood of $\tilde{x}_{0}$ in which the self-similar behavior is exhibited; and third, a self-similar profile based on which the self-similar solution is determined. In our computations, the center of self-similarity must be $\tilde{q}_{0}=(1,0)^{T}$, the location of the maximum vorticity, since this is the point at which the solution is about to blow up. To identify a "self-similar neighborhood" of $\tilde{q}_{0}$, we define, for each fixed time instant $t$, the neighborhood of the maximum vorticity,

$$
D_{\infty}(t)=\left\{(r, z) \in D\left(1, \frac{1}{4} L\right):|\omega(r, z, t)| \geq \frac{1}{2}\|\omega(\cdot, t)\|_{\infty}\right\}
$$

and plot in Figure 8(a) the boundary curves of $D_{\infty}(t)$,

$$
C_{\infty}(t)=\left\{(r, z) \in D\left(1, \frac{1}{4} L\right):|\omega(r, z, t)|=\frac{1}{2}\|\omega(\cdot, t)\|_{\infty}\right\},
$$

Copyright $@$ by SIAM. Unauthorized reproduction of this article is prohibited. 


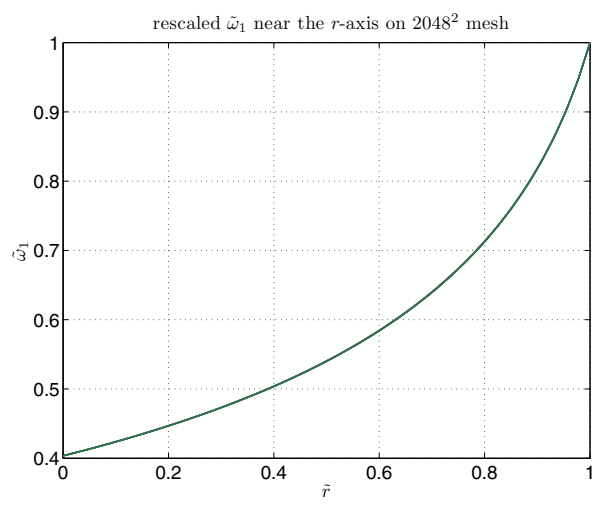

(a) near the $r$-axis

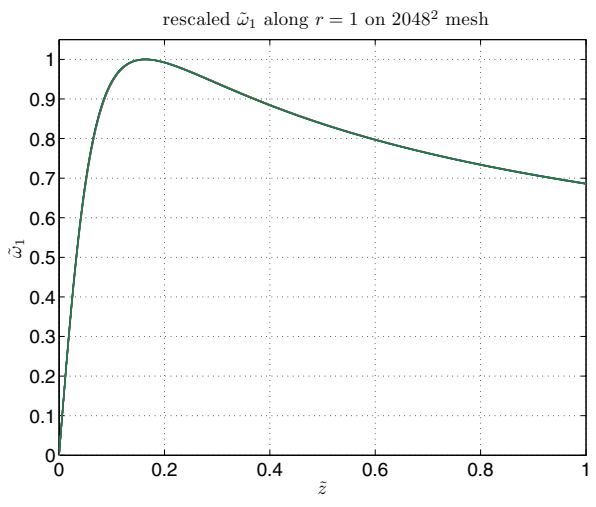

(b) along the wall $r=1$

Fig. 9 The $1 D$ self-similar profiles of $\omega_{1}$ (a) near the $r$-axis and (b) along the wall $r=1$, obtained by rescaling the solutions at the nine time instants given by (4.11).

at the nine time instants

$$
\begin{aligned}
& \mathcal{T}_{s}=\{0.00347,0.00348,0.00349,0.0035 \\
&0.003501,0.003502,0.003503,0.003504,0.003505\}
\end{aligned}
$$

It can be observed from the figure that the level curves $C_{\infty}(t)$ shrink rapidly toward $\tilde{q}_{0}$ and have very similar shapes at the first few time instants when they are still visible in the figure. To reveal more clearly the asymptotic behavior of $C_{\infty}(t)$ at the later times, we plot the same nine curves in Figure $8(\mathrm{~b})$ in log-log scale against the variables $(1-r)$ and $z$. The results show that the shape of $C_{\infty}(t)$ indeed remains roughly the same at all nine time instants. Motivated by this observation, we rescale each curve $C_{\infty}(t)$ according to the rule

$$
\tilde{r}=1-\frac{1-r}{d_{r}\left(C_{\infty}(t)\right)}, \quad \tilde{z}=\frac{z}{d_{z}\left(C_{\infty}(t)\right)},
$$

where

$$
d_{r}\left(C_{\infty}(t)\right)=\max _{(r, z) \in C_{\infty}(t)}|1-r|, \quad d_{z}\left(C_{\infty}(t)\right)=\max _{(r, z) \in C_{\infty}(t)}|z|
$$

The rescaled curves $\tilde{C}_{\infty}(t)$, as shown in Figure $8(\mathrm{c})$, collapse almost perfectly to a single curve, which confirms the existence of a self-similar neighborhood of $\tilde{q}_{0}$. The small variations among the different rescaled curves are shown in Figure 8(d), which can be viewed as manifestations of the local (inexact) nature of the self-similarity.

4.7.2. Existence of Self-Similar Profiles. By employing a procedure completely similar to that described in the previous section, we examine the solution $\left(u_{1}, \omega_{1}, \psi_{1}\right)$ in the self-similar neighborhood $D_{\infty}(t)$ and confirm the existence of self-similar profiles. For purposes of illustration, we plot in Figure 9 the 1D self-similar profiles of $\omega_{1}$ along selected 1D $r$ - and $z$-mesh lines, and in Figure 10 we show the 2D contour plots of $\omega_{1}$ near the location of the maximum vorticity at $t=0.0034$ and 0.003505 . Similar plots are also obtained for $u_{1}, \psi_{1}$ and are omitted here for the sake of brevity. 


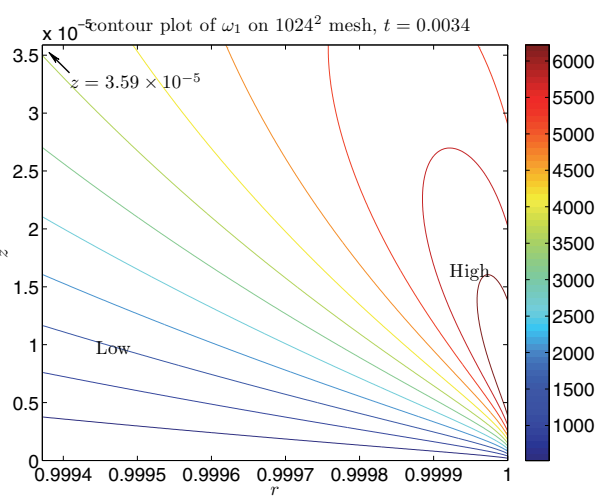

(a) $t=0.0034$

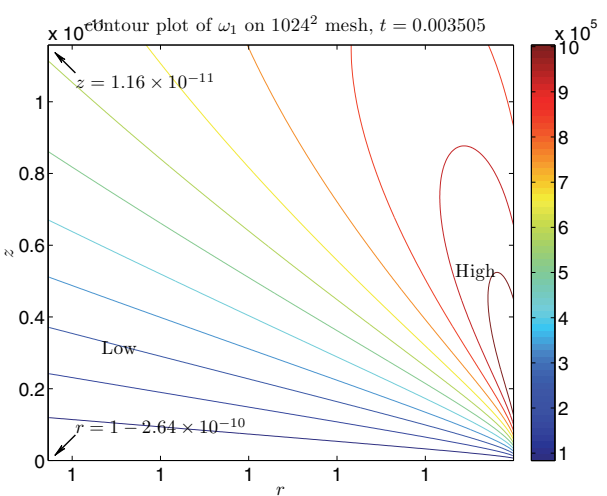

(b) $t=0.003505$

Fig. 10 The $2 D$ contour plot of $\omega_{1}$ near the location of the maximum vorticity at (a) $t=0.0034$ and (b) $t=0.003505$, both computed on the $1024 \times 1024$ mesh.

Table 13 Scaling exponents of the self-similar solution (4.8).

\begin{tabular}{cccccccc}
\hline Mesh size & $\hat{\gamma}_{l}$ & $\hat{\gamma}_{u}$ & $\hat{\gamma}_{\omega}$ & $\hat{\gamma}_{\psi}$ & $-1+\frac{1}{2} \hat{\gamma}_{l}$ & $-1+2 \hat{\gamma}_{l}$ & $\hat{\gamma}_{u}-\hat{\gamma}_{l}$ \\
\hline $1024 \times 1024$ & 2.7359 & 0.4614 & -0.9478 & 4.7399 & 0.3679 & 4.4717 & -2.2745 \\
$1280 \times 1280$ & 2.9059 & 0.4629 & -0.9952 & 4.8683 & 0.4530 & 4.8118 & -2.4430 \\
$1536 \times 1536$ & 2.9108 & 0.4600 & -0.9964 & 4.8280 & 0.4554 & 4.8215 & -2.4508 \\
$1792 \times 1792$ & 2.9116 & 0.4602 & -0.9966 & 4.8294 & 0.4558 & 4.8232 & -2.4514 \\
$2048 \times 2048$ & 2.9133 & 0.4604 & -0.9972 & 4.8322 & 0.4567 & 4.8266 & -2.4529 \\
\hline
\end{tabular}

4.7.3. Self-Similar Analysis. Given the existence of self-similar profiles in the self-similar neighborhood $D_{\infty}(t)$, we conclude that the solution $\left(u_{1}, \omega_{1}, \psi_{1}\right)$ develops a locally self-similar structure near the point of blowup $\tilde{q}_{0}$. This motivates the representation formula (4.8) with $\tilde{x}_{0}=\tilde{q}_{0}=(1,0)^{T}$. Upon the substitution of (4.8) into the $3 \mathrm{D}$ Euler equations (2.1), we obtain the dominant balance

$$
\begin{aligned}
\gamma_{u}-1 & =\gamma_{u}+\gamma_{\psi}-2 \gamma_{l}, \\
\gamma_{\omega}-1 & =\gamma_{\omega}+\gamma_{\psi}-2 \gamma_{l}=2 \gamma_{u}-\gamma_{l}, \\
\gamma_{\psi}-2 \gamma_{l} & =\gamma_{\omega},
\end{aligned}
$$

which, after simplification, yields the one-parameter family of scaling laws

$$
\gamma_{u}=-1+\frac{1}{2} \gamma_{l}, \quad \gamma_{\omega}=-1, \quad \gamma_{\psi}=-1+2 \gamma_{l} .
$$

Table 13 summarizes the scaling exponents estimated from the numerical data, where $\hat{\gamma}_{u}-\hat{\gamma}_{l}$ roughly equals $\gamma$, the scaling exponent of the maximum vorticity $\|\omega\|_{\infty}$ (cf. (4.2); recall that $\left.\|\omega\|_{\infty}=\left|u_{1, z}\left(\tilde{q}_{0}\right)\right|\right)$. It is clearly seen that (4.12) is approximately satisfied, which confirms the existence of a self-similar solution. In addition to (4.12), it is also known that, for a blowup to occur, the scaling exponent $\gamma_{l}$ must satisfy $\gamma_{l} \geq \frac{2}{5}$ in view of energy conservation [23]. If the velocity $u$ is uniformly bounded, which is the case in our computation, $\gamma_{l}$ must also satisfy $\gamma_{l} \geq 1$ [23]. It is clear from Table 13 that both constraints are satisfied by our numerical data. 
The leading-order equations resulting from the dominant balance take the form

$$
\begin{aligned}
-\gamma_{u} U+\gamma_{l}(\tilde{r}, \tilde{z}) \cdot \nabla U+\left[-\Psi_{\tilde{z}} U_{\tilde{r}}+\Psi_{\tilde{r}} U_{\tilde{z}}\right] & =0, \\
-\gamma_{\omega} \Omega+\gamma_{l}(\tilde{r}, \tilde{z}) \cdot \nabla \Omega+\left[-\Psi_{\tilde{z}} \Omega_{\tilde{r}}+\Psi_{\tilde{r}} \Omega_{\tilde{z}}\right] & =2 U U_{\tilde{z}}, \\
-\left[\Psi_{\tilde{r} \tilde{r}}+\Psi_{\tilde{z} \tilde{z}}\right] & =\Omega,
\end{aligned}
$$

where $(\tilde{r}, \tilde{z})$ are the rescaled coordinates. In principle, one can solve these equations numerically and compare the solutions to those obtained from rescaling (cf. (4.8)), which would provide additional supporting evidence for the existence of a self-similar solution. This has not been done in the current study, but may be considered in a future work.

Finally, the fitting results shown in Table 13 imply that

$$
\begin{gathered}
\omega^{r}=-r u_{1, z}=O\left(t_{s}-t\right)^{-2.45}, \quad \omega^{\theta}=r \omega_{1}=O\left(t_{s}-t\right)^{-1}, \\
\omega^{z}=2 u_{1}+r u_{1, r}=O\left(t_{s}-t\right)^{-2.45}
\end{gathered}
$$

which confirms the scaling law $\|\omega(\cdot, t)\|_{\infty}=O\left(t_{s}-t\right)^{-2.45}$ and hence the existence of a finite-time singularity.

4.8. Understanding the Blowup. For the specific initial condition (2.2a) considered in our study, it is observed that $r u_{1}^{0}$ is monotonically increasing in both $r$ and $z$ within the quarter cylinder $D\left(1, \frac{1}{4} L\right)$. It turns out that this property is preserved by equations (2.1) (for reasons yet to be determined); thus $u_{1, z}$ and consequently $\omega_{1}$ (cf. (2.1b)) remain positive for as long as the solution is smooth. The positivity of $\omega_{1}$ and the homogeneous boundary condition of $\psi_{1}$ together imply the positivity of $\psi_{1}$ (cf. (2.1c)), which in turn implies that

$$
u^{z}=2 \psi_{1}+r \psi_{1, r}=\psi_{1, r} \leq 0 \quad \text { on } \quad r=1, z \in\left[0, \frac{1}{4} L\right] .
$$

This shows that the flow has a compression mechanism near the corner $\tilde{q}_{0}=(1,0)^{T}$ (Figure 11(a); recall that $u^{z}$ is odd at $z=0$ ), which seems to be responsible for the generation of the finite-time singularity observed at $\tilde{q}_{0}$. Indeed, as far as the formation of a singularity is concerned, the precise form of the initial data seems to be immaterial. As long as $r u_{1}^{0}$ has the desired symmetry properties and is monotonically increasing in both $r$ and $z$ in the quarter cylinder $D\left(1, \frac{1}{4} L\right)$, the solution of the initialboundary value problem (2.1)-(2.2) should develop a singularity in finite time, in much the same way as the solution described in this paper does.

From a physical point of view, the blowup can be deduced from vorticity kinematics applied to the initially rotating eddy [19]. The gradient of circulation down the tube, $2 \pi r u_{z}^{\theta}$, creates a $\theta$-component of vorticity (cf. (2.1b)). This component in turn creates the flow $\left(u^{r}, u^{z}\right)$ (cf. (2.1c)-(2.1d)) which advects toward the symmetry plane $z=0$ on the solid wall $r=1$. Since vortex lines threading through the wall are carried by this flow, their points of intersection with the wall move toward the symmetry plane $z=0$ and then collapse onto $z=0$ in finite time (see Figure 11(b)). This is similar to what was observed by [19] in the study of a model problem, which was derived as the leading-order approximation to a stretched version of the TaylorGreen initial value problem for the 3D Euler equations. The model closely resembles the axisymmetric Euler equations, except that the fluid inertia $\left(D_{t} u^{r}\right)$ in the radial transport equation is missing. Since the variable $u^{\theta}$ studied in [19] occurs as coefficients of the asymptotic expansions, the blowup of its $z$-derivatives merely indicates 


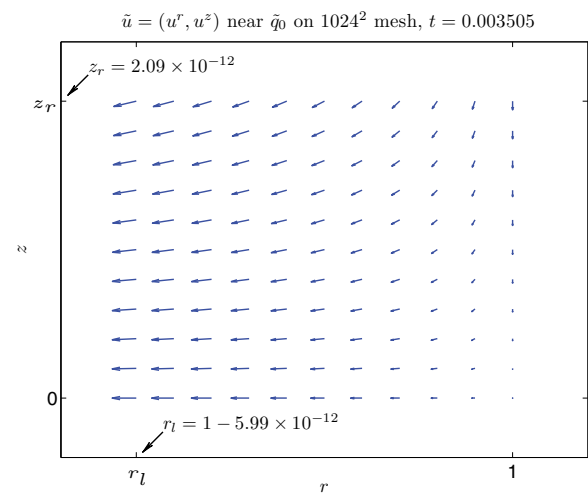

(a) local flow field

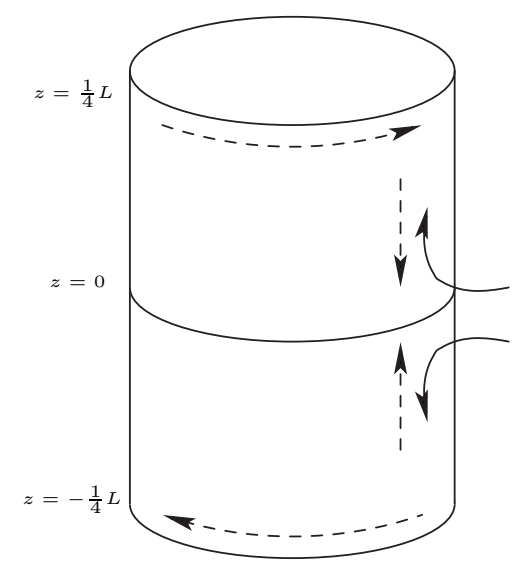

(b) global vorticity dynamics

Fig. II Understanding the blowup: (a) local velocity field near the point of the maximum vorticity; (b) global vorticity kinematics of the $3 D$ Euler singularity. In (b), the vortex lines (curved solid arrows) end at the wall and are brought to sections of zero circulation by the axial flow (straight dashed arrows). The curved dash arrows indicate vortical circulation. See also Figure 5 in [19] and Figure 7 in [20].

the breakdown of the expansions and the return of the flow to three-dimensionality. It does not imply the loss of regularity of the underlying solutions.

Despite the apparent similarity between our computations and the model studied by [19], there is a fundamental difference between the two scenarios. More precisely, in [19], it was observed that the absence of radial momentum transfer creates a favorable pressure gradient, which sets up an axial flow near the solid wall toward the symmetry plane $z=0$. In our case, however, it is observed that the pressure gradient near the solid wall $r=1$ and the symmetry plane $z=0$ is unfavorable, in the sense that it tends to push fluids away from $z=0$ (Figure 12). Thus unlike the scenario described in [19], it must be the radial fluid inertia, not the pressure gradient, that is responsible for the finite-time blowup observed at the corner $\tilde{q}_{0}=(1,0)^{T} \cdot{ }^{12}$

4.9. A ID Model. Motivated by the observation that the Euler singularity is likely a consequence of a compression flow along the solid wall, we propose a 1D model

$$
\begin{aligned}
\theta_{t}+v \theta_{z} & =0, \quad z \in(0, L), \\
\omega_{t}+v \omega_{z} & =\theta_{z},
\end{aligned}
$$

where the nonlocal velocity $v$ satisfies the Biot-Savart law

$$
v(z)=\frac{1}{\pi} \int_{0}^{L} \omega(y) \log |\sin [\mu(z-y)]| d y, \quad \mu=\pi / L .
$$

Heuristically, (4.13a)-(4.13b) can be obtained directly from their full 3D counterparts (2.1a)-(2.1b), by restricting the latter equations to the solid wall $r=1$, by observing

\footnotetext{
${ }^{12}$ There exist, however, interesting alternative views which attribute the formation of the singularity to the mismatch of the second derivatives $p_{r r}$ and $p_{z z}$ of the pressure $p$ at the point of the singularity; see the very recent work of [3] for details.
} 


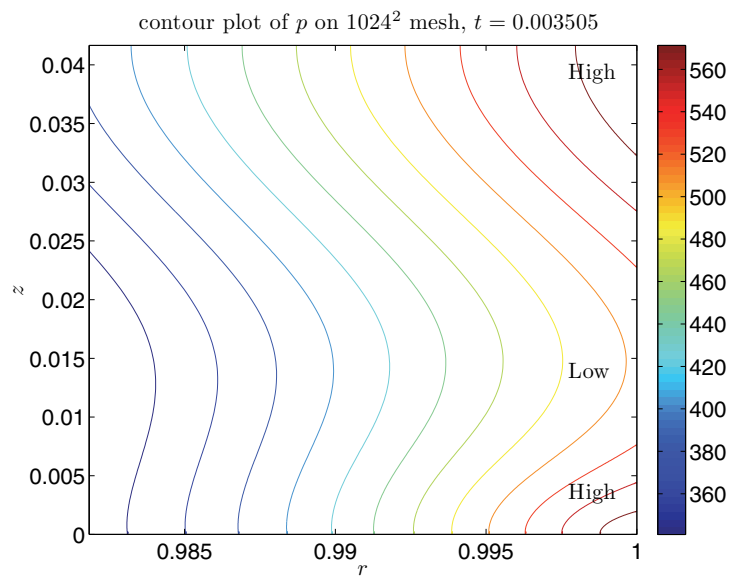

Fig. 12 The contour plot of the pressure $p$ near the location of the maximum vorticity at $t=$ 0.003505. Note the unfavorable axial pressure gradient near $z=0$.

(cf. $(2.2 \mathrm{c}))$

$$
\left.\psi_{1}\right|_{r=1}=0,\left.\quad u^{r}\right|_{r=1}=-\left.r \psi_{1, z}\right|_{r=1}=0,
$$

and then by introducing the identifications

$$
\theta(z) \sim u_{1}^{2}(1, z), \quad \omega(z) \sim \omega_{1}(1, z), \quad v(z) \sim u^{z}(1, z)=\psi_{1, r}(1, z) .
$$

The Biot-Savart law (4.13c), on the other hand, can be (formally) derived from its full $3 \mathrm{D}$ analogue, by observing that $\omega_{1}$ is essentially supported in a narrow strip near the solid wall $r=1$, and by assuming that it is approximately constant in $r$ in its support (see [21] for details). ${ }^{13}$ For the initial condition

$$
\theta^{0}(z)=10^{4} \sin ^{2}\left(\frac{2 \pi}{L} z\right), \quad \omega^{0}(z)=0
$$

which is simply the restriction of (2.2a) to $r=1$, the solution of (4.13) is observed to exhibit singular behaviors in finite time, in much the same way as the singular solution described in this paper does (Figure 13). Motivated by these observations, we prove, together with our collaborators, in [21] the existence of a finite-time singularity for this $1 \mathrm{D}$ model, where the initial data leading to the blowup have symmetry properties completely analogous to those possessed by (4.13d). This provides further supporting evidence for the existence of an Euler singularity, and the generalization of the argument to the full 3D case offers an interesting direction for future study.

5. Conclusions and Future Work. In this paper, we have numerically studied the 3D axisymmetric Euler equations in a periodic cylinder and have discovered a class of potentially singular solutions from carefully chosen initial data. By employing a specially designed yet highly effective adaptive mesh, we have resolved the nearly singular solution with high accuracy and have advanced the solution to a point asymptotically close to the predicted singularity time. Detailed analysis based on rigorous

\footnotetext{
${ }^{13}$ Although $\omega_{1}$ is not really nearly constant in $r$ near the solid wall $r=1$, numerical studies show that solutions of (4.13) do exhibit behaviors very similar to those observed in solutions of (2.1); see below.
} 


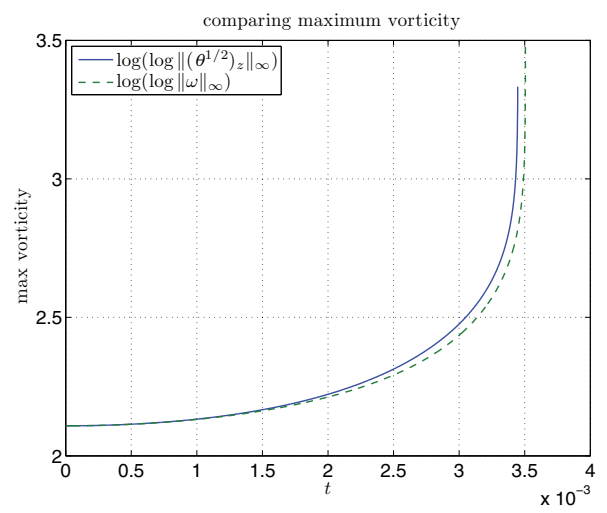

(a) $\log \left(\log \left\|\left(\theta^{1 / 2}\right)_{z}\right\|_{\infty}\right)$ vs. $\log \left(\log \|\omega\|_{\infty}\right)$

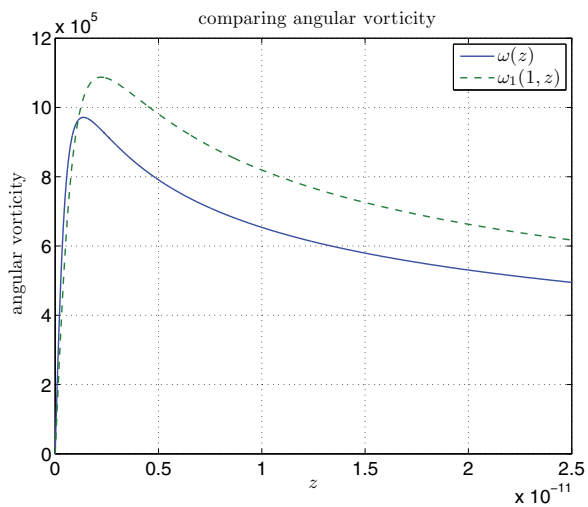

(c) $\omega(z)$ vs. $\omega_{1}(1, z)$

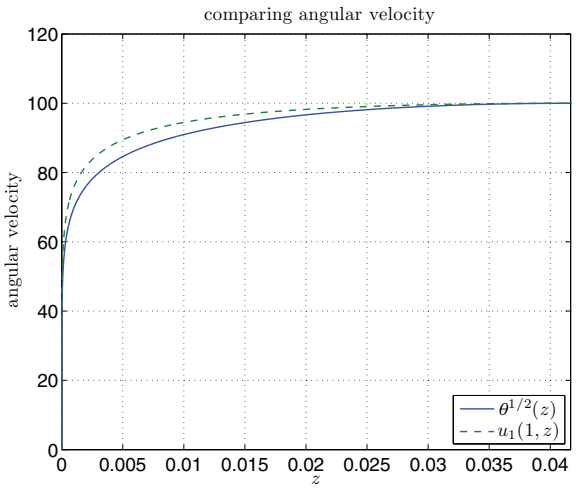

(b) $\theta^{1 / 2}(z)$ vs. $u_{1}(1, z)$

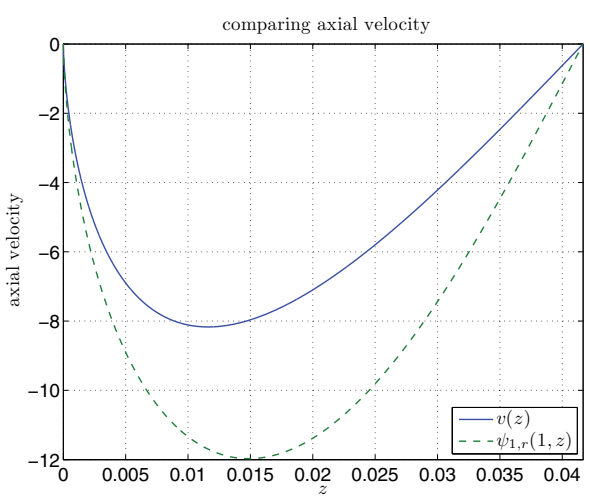

(d) $v(z)$ vs. $\psi_{1, r}(1, z)$

Fig. 13 Comparison of numerical solutions of the $1 D$ model (4.13) with those of the $3 D$ axisymmetric Euler equations (2.1): (a) maximum vorticity, (b) angular velocity, (c) angular vorticity, and (d) axial velocity. In all the plots the solution of the $1 D$ model is computed at $t=0.003447$ and that of the $3 D$ Euler is computed at $t=0.003505$.

mathematical blowup/nonblowup criteria, including Beale-Kato-Majda, ConstantinFefferman-Majda, and Deng-Hou-Yu, provides convincing evidence for the existence of a singularity. Local analysis near the point of the singularity also reveals a selfsimilar structure in the blowup, as well as a 1D model which is seen to capture the essential features of the singular solutions along the solid wall, and for which existence of finite-time singularities can be established rigorously.

Besides providing a promising candidate for the finite-time blowup of the 3D Euler equations, our computations also suggest a possible route to the finite-time blowup of the 2D Boussinesq equations. The Boussinesq equations describe the motion of variable-density, stratified flows under the influence of gravitational forces, and like the 3D Euler equations, the existence or nonexistence of globally regular solutions to the 2D Boussinesq equations is a well-known open problem in fluid dynamics (see, for example, [67]). Since the 2D Boussinesq equations are known to be qualitatively similar to the 3D axisymmetric Euler equations away from the symmetry axis [59], and since the singularity discovered in our Euler computations lies right on the solid boundary, the solution of the 2D Boussinesq equations resulting from similar initial 
data is expected to develop a singularity in finite time, in much the same way as the solution described in this paper does. The existence of such a singularity has been confirmed numerically in a separate computation (unpublished), and it can also be partly justified by the analysis of the 1D model (4.13), as suggested in [21], as well as by that of other related models as suggested in [52]. Very recently, the finite-time, self-similar blowup of a De Gregorio model for the 3D Euler equations and that of the 1D model (4.13) are established using a computer-assisted approach [18]. It is hoped that the same idea can be applied to the 3D Euler and 2D Boussinesq equations, and that the existence of a finite-time singularity, as suggested by this work, can be established rigorously.

Acknowledgments. This research was supported in part by NSF FRG grant DMS-1159138 and NSF grants DMS-1613861, DMS-1907977, and DMS-1912654. The authors would like to gratefully acknowledge the computing resources provided by the SHC cluster at Caltech Center for Advanced Computing Research (CACR) and the Brutus cluster at ETH Zürich (ETHZ). The authors gratefully acknowledge the excellent support provided by the staff members at SHC, especially Sharon Brunett, and the support provided by Prof. Petros Koumoutsakos at ETHZ, who kindly allowed them to use his computing resources. The authors also thank the anonymous referees for their helpful comments. The first author gratefully acknowledges the travel support provided by NSF FRG grant DMS-1159133, made available to him by Prof. Alexander Kiselev, for his trip to 2013 Stanford summer school, and by the Department of Computing and Mathematical Sciences at Caltech, for his trip to 2013 AMS Fall central sectional meeting at Washington University in St. Louis.

\section{REFERENCES}

[1] M. Arioli, J. W. Demmel, And I. S. Duff, Solving sparse linear systems with sparse backward error, SIAM J. Matrix Anal. Appl., 10 (1989), pp. 165-190, https://doi.org/10.1137/ 0610013. (Not cited)

[2] C. Bardos AND E. S. Titi, Euler equations for incompressible ideal fluids, Russian Math. Surveys, 62 (2007), pp. 409-451. (Cited on pp. 794, 797)

[3] D. Barkley, A Fluid Mechanic's Analysis of the Teacup Singularity, preprint, https://arxiv. org/abs/1902.05993, 2019. (Cited on p. 829)

[4] J. T. BEAle, T. KATO, AND A. MAJDA, Remarks on the breakdown of smooth solutions for the 3-D Euler equations, Comm. Math. Phys., 94 (1984), pp. 61-66. (Cited on pp. 795, 809, 816)

[5] M. Berger and R. V. Kohn, A rescaling algorithm for the numerical calculation of blowing-up solutions, Comm. Pure Appl. Math., 41 (1988), pp. 841-863. (Cited on p. 801)

[6] M. Berger and J. Oliger, Adaptive mesh refinement for hyperbolic partial differential equations, J. Comput. Phys., 53 (1984), pp. 484-512. (Cited on p. 801)

[7] O. N. Boratav and R. B. Pelz, Direct numerical simulation of transition to turbulence from a high-symmetry initial condition, Phys. Fluids, 6 (1994), pp. 2757-2784. (Cited on pp. 796, 819)

[8] C. J. Budd, S. Chen, And R. D. Russell, New self-similar solutions of the nonlinear Schrödinger equation with moving mesh computations, J. Comput. Phys., 152 (1999), pp. 756789. (Cited on p. 801)

[9] C. J. Budd, W. Huang, and R. D. Russell, Moving mesh methods for problems with blow-up, SIAM J. Sci. Comput., 17 (1996), pp. 305-327, https://doi.org/10.1137/ S1064827594272025. (Cited on p. 801)

[10] M. D. Bustamante And R. M. KerR, 3D Euler about a $2 D$ symmetry plane, Phys. D, 237 (2008), pp. 1912-1920. (Cited on pp. 796, 809)

[11] L. Caffarelli, R. Kohn, and L. Nirenberg, Partial regularity of suitable weak solutions of the Navier-Stokes equations, Comm. Pure Appl. Math., 35 (1982), pp. 771-831. (Cited on p. 797)

Copyright $@$ by SIAM. Unauthorized reproduction of this article is prohibited. 
[12] R. E. CAflisch, Singularity formation for complex solutions of the $3 D$ incompressible Euler equations, Phys. D, 67 (1993), pp. 1-18. (Cited on p. 796)

[13] D. ChaE, Nonexistence of self-similar singularities for the 3D incompressible Euler equations, Comm. Math. Phys., 273 (2007), pp. 203-215. (Cited on p. 824)

[14] D. ChaE, On the generalized self-similar singularities for the Euler and the Navier-Stokes equations, J. Funct. Anal., 258 (2010), pp. 2865-2883. (Cited on p. 824)

[15] D. ChaE, On the self-similar solutions of the 3D Euler and the related equations, Comm. Math. Phys., 305 (2011), pp. 333-349. (Cited on p. 824)

[16] D. Chae, P. Constantin, and J. Wu, An incompressible $2 D$ didactic model with singularity and explicit solutions of the $2 D$ Boussinesq equations, J. Math. Fluid Mech., 16 (2014), pp. 473-480. (Cited on p. 797)

[17] D. Chae And T.-P. Tsai, Remark on Luo-Hou's ansatz for a self-similar solution to the $3 D$ Euler equations, J. Nonlinear Sci., 25 (2015), pp. 193-202. (Cited on p. 824)

[18] J. Chen, T. Y. Hou, And D. Huang, On the Finite Time Blowup of the De Gregorio Model for the 3D Euler Equation, preprint, https://arxiv.org/abs/1905.06387, 2019. (Cited on p. 832)

[19] S. Childress, Nearly two-dimensional solutions of Euler's equations, Phys. Fluids, 30 (1987), pp. 944-953. (Cited on pp. 828, 829)

[20] S. ChILdress, Topological Fluid Dynamics for Fluid Dynamicists, preprint, https://www.math. nyu.edu/faculty/childres/tfd.pdf, 2004. (Cited on p. 829)

[21] K. Choi, T. Hou, A. Kiselev, G. Luo, V. Sverak, and Y. Yao, On the finite-time blowup of a $1 D$ model for the $3 D$ axisymmetric Euler equations, Comm. Pure Appl. Math., 70 (2017), pp. 2218-2243. (Cited on pp. 798, 830, 832)

[22] P. G. Ciarlet, The Finite Element Method for Elliptic Problems, Classics Appl. Math. 40, SIAM, Philadelphia, 2002, https://doi.org/10.1137/1.9780898719208. (Not cited)

[23] P. Constantin, Geometric statistics in turbulence, SIAM Rev., 36 (1994), pp. 73-98, https:// doi.org/10.1137/1036004. (Cited on pp. 820, 827)

[24] P. Constantin, On the Euler equations of incompressible fluids, Bull. Amer. Math. Soc. (N.S.), 44 (2007), pp. 603-621. (Cited on pp. 794, 797)

[25] P. Constantin, C. Fefferman, And A. Majda, Geometric constraints on potentially singular solutions for the 3-D Euler equations, Comm. Partial Differential Equations, 21 (1996), pp. 559-571. (Cited on pp. 795, 821)

[26] J. Deng, T. Y. Hou, And X. Yu, Geometric properties and nonblowup of $3 D$ incompressible Euler flow, Comm. Partial Differential Equations, 30 (2005), pp. 225-243. (Cited on pp. 795,821$)$

[27] W. E And C.-W. Shu, Small-scale structures in Boussinesq convection, Phys. Fluids, 6 (1994), pp. 49-58. (Cited on p. 796)

[28] T. M. Elgindi And I.-J. Jeong, Finite-Time Singularity Formation for Strong Solutions to the Boussinesq System, preprint, https://arxiv.org/abs/1708.02724, 2017. (Cited on p. 798)

[29] T. M. Elgindi And I.-J. Jeong, Finite-Time Singularity Formation for Strong Solutions to the Axi-symmetric 3D Euler Equations, preprint, https://arxiv.org/abs/1802.09936, 2018. (Cited on p. 798)

[30] T. M. Elgind, Finite-Time Singularity Formation for $C^{1, \alpha}$ Solutions to the Incompressible Euler Equations on $\mathbb{R}^{3}$, preprint, https://arxiv.org/abs/1904.04795, 2019. (Cited on p. 798)

[31] C. L. Fefferman, Existence and smoothness of the Navier-Stokes equation, in The Millennium Prize Problems, J. Carlson, A. Jaffe, and A. Wiles, eds., Clay Mathematics Institute, Cambridge, MA, 2006, pp. 57-67; also available online from http://www.claymath.org/ library/monographs/MPPc.pdf. (Cited on pp. 795, 797)

[32] A. B. FERrARI, On the blow-up of solutions of the 3-D Euler equations in a bounded domain, Comm. Math. Phys., 155 (1993), pp. 277-294. (Cited on pp. 795, 816)

[33] H. FujitA, On the blowing up of solutions of the Cauchy problem for $u_{t}=\Delta u+u^{1+\alpha}$, J. Fac. Sci. Univ. Tokyo Sect. A Math., 16 (1966), pp. 105-113. (Cited on p. 800)

[34] J. D. GibBon, The three-dimensional Euler equations: Where do we stand?, Phys. D, 237 (2008), pp. 1894-1904. (Cited on pp. 794, 796)

[35] J. D. Gibbon, D. R. Moore, and J. T. Stuart, Exact, infinite energy, blow-up solutions of the three-dimensional Euler equations, Nonlinearity, 16 (2003), pp. 1823-1831. (Cited on p. 794)

[36] J. D. Gibbon and E. S. Titi, The 3D incompressible Euler equations with a passive scalar: A road to blow-up?, J. Nonlinear Sci., 23 (2013), pp. 993-1000. (Cited on p. 795)

[37] R. Grauer, C. Marliani, and K. Germaschewski, Adaptive mesh refinement for singular solutions of the incompressible Euler equations, Phys. Rev. Lett., 80 (1998), pp. 4177-4180. (Cited on p. 819)

Copyright $@$ by SIAM. Unauthorized reproduction of this article is prohibited. 
[38] R. Grauer and T. C. Sideris, Numerical computation of $3 D$ incompressible ideal fluids with swirl, Phys. Rev. Lett., 67 (1991), pp. 3511-3514. (Cited on p. 796)

[39] B. Gustafsson, H.-O. Kreiss, and J. Oliger, Time Dependent Problems and Difference Methods, Wiley-Interscience, New York, 1995. (Cited on p. 805)

[40] P. Hénon, P. Ramet, and J. Roman, PaStiX: A high-performance parallel direct solver for sparse symmetric definite systems, Parallel Comput., 28 (2002), pp. 301-321. (Not cited)

[41] K. HölliG, Finite Element Methods with B-Splines, SIAM, Philadelphia, 2003, https://doi. org/10.1137/1.9780898717532. (Cited on pp. 803, 804)

[42] T. Y. HOU AND Z. LEI, On the stabilizing effect of convection in three-dimensional incompressible flows, Comm. Pure Appl. Math., 62 (2009), pp. 501-564. (Cited on p. 797)

[43] T. Y. Hou And C. M. LI, Dynamic stability of the three-dimensional axisymmetric NavierStokes equations with swirl, Comm. Pure Appl. Math., 61 (2008), pp. 661-697. (Cited on pp. 797, 799)

[44] T. Y. Hou AND R. LI, Dynamic depletion of vortex stretching and non-blowup of the 3-D incompressible Euler equations, J. Nonlinear Sci., 16 (2006), pp. 639-664. (Cited on pp. 796, 809)

[45] T. Y. Hou And R. LI, Blowup or no blowup? The interplay between theory and numerics, Phys. D, 237 (2008), pp. 1937-1944. (Cited on pp. 796, 819)

[46] W. Huang, J. Ma, And R. D. Russell, A study of moving mesh PDE methods for numerical simulation of blowup in reaction diffusion equations, J. Comput. Phys., 227 (2008), pp. 6532-6552. (Cited on p. 801)

[47] W. Huang, Y. Ren, And R. D. Russell, Moving mesh methods based upon moving mesh partial differential equations, J. Comput. Phys., 113 (1994), pp. 279-290. (Cited on p. 801)

[48] Y. Huang And A. L. Bertozzi, Self-similar blowup solutions to an aggregation equation in $R^{n}$, SIAM J. Appl. Math., 70 (2010), pp. 2582-2603, https://doi.org/10.1137/090774495. (Cited on p. 800)

[49] T. Kato, Nonstationary flows of viscous and ideal fluids in $R^{3}$, J. Funct. Anal., 9 (1972), pp. 296-305. (Cited on p. 795)

[50] R. M. KERR, Evidence for a singularity of the three-dimensional incompressible Euler equations, Phys. Fluids A, 5 (1993), pp. 1725-1746. (Cited on pp. 796, 809, 819)

[51] R. M. KerR, Bounds for Euler from vorticity moments and line divergence, J. Fluid Mech., 729 (2013), art. R2. (Cited on p. 796)

[52] A. KISELEv, Small scales and singularity formation in fluid dynamics, in Proceedings of the International Congress of Mathematicians, Rio de Janeiro, Brazil, 2018, pp. 2329-2356. (Cited on pp. 798, 832)

[53] A. Kiselev, L. Ryzhik, Y. Yao, and A. Zlatos, Finite time singularity for the modified SQG patch equation, Ann. of Math. (2), 184 (2016), pp. 909-948. (Cited on p. 798)

[54] A. Kiselev And V. Sverak, Small scale creation for solutions of the incompressible twodimensional Euler equation, Ann. of Math. (2), 180 (2014), pp. 1205-1220. (Cited on p. 798)

[55] A. LARios And E. S. Titi, Global regularity versus finite-time singularities: Some paradigms on the effect of boundary conditions and certain perturbations, in Recent Progress in the Theory of the Euler and Navier-Stokes Equations, London Math. Soc. Lecture Notes 430, J. C. Robinson, J. L. Rodrigo, W. Sadowski, and A. Vidal-Lopez, eds., Cambridge University Press, Cambridge, UK, 2016, pp. 96-125. (Cited on p. 798)

[56] J.-G. LiU AND W.-C. WANG, Convergence analysis of the energy and helicity preserving scheme for axisymmetric flows, SIAM J. Numer. Anal., 44 (2006), pp. 2456-2480, https://doi.org/ 10.1137/050639314. (Cited on p. 799)

[57] G. Luo And T. Y. Hou, Potentially singular solutions of the $3 D$ axisymmetric Euler equations, Proc. Natl. Acad. Sci. USA, 111 (2014), pp. 12968-12973. (Cited on pp. 797, 798)

[58] G. Luo And T. Y. Hou, Toward the finite-time blowup of the $3 D$ axisymmetric Euler equations: A numerical investigation, Multiscale Model. Simul., 12 (2014), pp. 1722-1776, https:// doi.org/10.1137/140966411. (Cited on pp. 797, 798, 802, 803, 804, 807, 810, 817, 819, 821)

[59] A. Majda And A. Bertozzi, Vorticity and Incompressible Flow, Cambridge University Press, Cambridge, UK, 2002. (Cited on pp. 797, 799, 831)

[60] D. W. Mclaughlin, G. C. Papanicolaou, C. Sulem, and P. L. Sulem, Focusing singularity of the cubic Schrödinger equation, Phys. Rev. A, 34 (1986), pp. 1200-1210. (Cited on p. 800)

[61] P. Orlandi and G. F. Carnevale, Nonlinear amplification of vorticity in inviscid interaction of orthogonal Lamb dipoles, Phys. Fluids, 19 (2007), art. 057106. (Cited on p. 819)

[62] G. Ponce, Remarks on a paper by J. T. Beale, T. Kato, and A. Majda, Comm. Math. Phys., 98 (1985), pp. 349-353. (Cited on p. 795)

Copyright (c) by SIAM. Unauthorized reproduction of this article is prohibited. 
[63] A. Pumir And E. D. Siggia, Development of singular solutions to the axisymmetric Euler equations, Phys. Fluids A, 4 (1992), pp. 1472-1491. (Cited on p. 796)

[64] T. Shirota and T. Yanagisawa, A continuation principle for the 3-D Euler equations for incompressible fluids in a bounded domain, Proc. Japan Acad. Ser. A Math. Sci., 69 (1993), pp. 77-82. (Cited on pp. 795, 816)

[65] M. Siegel AND R. E. Caflisch, Calculation of complex singular solutions to the $3 D$ incompressible Euler equations, Phys. D, 238 (2009), pp. 2368-2379. (Cited on p. 796)

[66] E. M. Stein, Singular Integrals and Differentiability Properties of Functions, Princeton University Press, Princeton, NJ, 1970. (Cited on p. 795)

[67] V. I. Yudovich, Eleven great problems of mathematical hydrodynamics, Mosc. Math. J., 3 (2003), pp. 711-737. (Cited on p. 831)

Copyright (c) by SIAM. Unauthorized reproduction of this article is prohibited. 